\title{
Intra-grain orientation distributions in hot-deformed aluminium: Orientation dependence and relation to deformation mechanisms
}

\author{
R. Quey ${ }^{\mathrm{a}, 1}$, J.H. Driver ${ }^{\mathrm{a}}$, P.R. Dawson ${ }^{\mathrm{b}}$ \\ ${ }^{a}$ École des Mines de Saint-Étienne, CNRS UMR 5307, 158 cours Fauriel, 42023 Saint-Étienne, Cedex 2, France \\ ${ }^{b}$ Sibley School of Mechanical and Aerospace Engineering, Cornell University, Ithaca, NY 14853, USA
}

\begin{abstract}
The development of intra-grain orientation distributions is analysed for 92 individual grains of an aluminium polycrystal deformed in plane strain compression at $400{ }^{\circ} \mathrm{C}$ to an effective strain of $\varepsilon=1.2$. Orientations of the crystallographic lattice were measured by using a microtexture tracking technique that combines deforming a split sample with collecting electron backscattering diffraction (EBSD) data on the internal surface at successively greater strains. In these experiments, more than 1000 orientations were taken within each of the grains at strains $0.19,0.42,0.77$ and 1.2, as well as in the initial, undeformed state. A high-resolution finite element simulation (1000 elements per grain on average) was conducted on a polycrystal whose grains were assigned the experimental orientations. Attributes of the orientation distributions were extracted from both the experimental and simulation data, including the average disorientation angles and the preferential disorientation axes. For both experiment and simulation, the average disorientation angles were found to increase up to $\varepsilon=0.5$ and then to saturate to values of $7-8^{\circ}$. It is shown that the preferential disorientation axes are distributed about the transverse direction (TD) up to $\varepsilon=0.5$ and then migrate at large strains to directions between the rolling (extension) direction (RD) and the normal (compression) direction (ND). Detailed crystal plasticity analyses show that the distribution of preferential disorientation axes is related to two mechanisms: (i) the development of anisotropy in the orientation distributions due to the accentuated activity of particular slip systems from stress heterogeneities and (ii) the peak-shifting transformation of the distribution arising from gradients of the reorientation velocity field imposed by the deformation.
\end{abstract}

Key words: Microtexture, Crystal plasticity, EBSD, Finite element method, Aluminium

\section{Introduction}

The intra-grain orientation distributions that develop in plastically deformed polycrystals have a significant influence on both mechanical strength [1] and subsequent softening phenomena during annealing, e.g. recrystallization nucleation [2]. The distributions can also provide important information on local deformation mechanisms, as the orientation spreading is directly related to

Email addresses: romain.quey@mines-stetienne.fr (R. Quey), julian.driver@mines-stetienne.fr (J.H. Driver), paul .dawson@cornell . edu (P.R. Dawson)

Preprint submitted to Journal of the Mechanics and Physics of Solids

July 20, 2015 
the crystal deformation mechanisms and grain interactions. Consequently, considerable attention has been devoted to the evolution of local disorientations that develop in polycrystals deformed under cold and hot-working conditions. In general, it has been observed that cold deformation leads to a continuous increase of average disorientation $(\Delta \theta)$ with strain $(\varepsilon)$ according to a power law relation $\Delta \theta \propto \varepsilon^{n}$ with $n \simeq 0.5$ [3, 4, 5]. However, for conventional shaping operations, hot deformation leads to a saturation disorientation of order $3-5^{\circ}$ at strains of $0.5-1$ due to dynamic recovery [6, 7, 8]. This type of average disorientation analysis has also been extended to disorientation distributions within individual grains in single crystals [9, 10] and large-grained polycrystals [11]. Glez and Driver [9, 10] analysed the orientation distributions in three stable-orientation single crystals of Al-1 wt.\%Mn deformed in hot plane strain compression. The analysis confirmed that the disorientation distributions develop rapidly at the beginning of deformation $(\varepsilon<0.5)$, but then tend to saturate at large strains.

In comparison to the considerable interest in disorientation angles, relatively little attention has been paid to the disorientation axes. More precise knowledge of the disorientation axes, however, could provide a better understanding of the dislocation structure and its evolution with plastic strain and/or deformation temperature. In the single crystal studies of Glez and Driver [9, 10], a distribution of the disorientation axes about the sample transverse direction (TD) was found under plane strain compression, which tended to weaken with strain. Later, Pantleon and coauthors [12, 13] analysed the orientation distributions of 45 grains of an aluminium polycrystal cold rolled to a total strain of 0.5. A disorientation axis about TD was also found, but with no obvious relationship between the disorientation properties and the grain orientations. While these studies, as well as similar investigations on copper [14], confirmed the presence of a preferential disorientation axis, to date, very few interpretations have been provided, especially in terms of any relationships between disorientation axes and the underlying deformation mechanisms. Tóth and coauthors [15] proposed a model for predicting the (pixel-to-pixel) disorientation axes observed in a copper polycrystal deformed by equal-channel angular pressing, but as this deformation mode includes a non-zero net rigid-body rotation, it is unclear whether the experimental results or the model are relevant in the context of plane strain compression.

In a previous publication [16], a new method was presented for tracking the lattice rotations of individual grains of a polycrystal deformed in plane strain compression to large strains. The method consisted of deforming a split sample with periodic interruptions in the loading to measure the local rotations of the crystal lattice within grains visible on the sample's internal surface by EBSD. A set of 187 grains in an aluminium sample were tracked to a strain of 1.2. It was shown that $80-90 \%$ of the grains exhibit a "unimodal" rotation, described by an average rotation and an orientation distribution about the average orientation, while the rest of the grains undergo orientation fragmentation, also called coarse deformation banding [16]. Deformation banding is used here in the classical sense introduced by Barrett [17] and developed for example by Humphreys and Hatherly [18]: volumes within a grain of roughly constant lattice orientation, with that orientation being significantly different from lattice orientations present elsewhere in that grain. This definition implies multimodal rotations. In a later publication [19], the average lattice rotations were compared to crystal plasticity predictions with good qualitative agreement. For the grains undergoing orientation fragmentation, the experimental data were complemented by a finite element simulation of a virtual polycrystal undergoing the same plane strain compression defor- 
mation. For both experiment and simulation, it was found that orientation fragmentation arises in crystals with lattice orientations aligning symmetrically with the loading axes and possessing unstable reorientation velocity conditions [20].

In the present work, the orientation distributions of only the grains undergoing unimodal rotations are analysed with particular emphasis on the disorientation axes developed within each grain. Lattice disorientation distributions extracted from crystal plasticity simulations are compared to the distributions measured using EBSD at several strain levels. The paper proceeds as follows. First, the underlying principles for the microtexture tracking experiment and for the finite element simulation are summarized in Section 2. Next, we review the essential metrics for the orientation distributions in Section 3 and present the metrics of the experimental and simulation distributions in Section 4 . Section 5 then focuses on relating the metrics, especially the preferential disorientation axes, to the local deformations. Sections 6 and 7 complete the paper with discussion of the results and conclusions.

\section{Methodology}

Both experiment and simulation are employed to analyse the evolution of orientation distributions of individual grains of an aluminium polycrystal deformed to large strain by hot plane strain compression. The experiment is based on the microtexture tracking technique [16], previously employed to analyse the average grain rotations [16, 19], orientation fragmentation [20] and subgrain evolutions [11]. The simulation is carried out using the crystal plasticity finite element method with finely discretized grains, and is essentially the same as that used for the orientation fragmentation analysis [20]. Brief overviews of the two methods are provide here; the reader is referred to Refs. [16, 21, 22] for additional details.

\subsection{Experiment}

The "microtexture tracking" technique consists of following the orientations of individual grains of a polycrystal on the internal surface of a split sample subject to successive plastic deformations [16], as pioneered by Barrett and Levenson [23] and Panchanadeeswaran et al. [24]. A high-purity Al-0.1 wt. $\% \mathrm{Mn}$ alloy with an average grain size of about $300 \mu \mathrm{m}$ and a weak initial crystallographic texture was used. A split sample was fabricated of two identical, $8 \times 3.5 \times 10 \mathrm{~mm}$ parts. The sample directions correspond to RD, TD and ND, respectively. The full sample was made by assembling the two parts along their RD-ND faces. The sample was subjected to plane strain compression by compressing it along ND and extending it along RD. Prior to deformation, an orientation map of the grain structure was constructed by EBSD on a $4 \times 4 \mathrm{~mm}$ region located at the centre of one of the two sample parts, using a step size of $5 \mu \mathrm{m}$. The sample was then deformed at $400{ }^{\circ} \mathrm{C}$ to successive strains of $0.19,0.42,0.77$ and 1.2. After each deformation step, the sample was quenched to retain the "hot deformation" microstructure. As described in Refs. [16, 11], the material does not recrystallize during successive deformations at $400{ }^{\circ} \mathrm{C}$. Without any additional polishing, orientation maps were measured at each strain level on the same area of the sample, using the same spatial resolution. A schematic of the experimental set-up together with orientation maps of the microstructures obtained at successive strain levels can be found in Refs. [16, 20]. It was previously shown that tracking the grain rotations on the internal surface of a split sample does 
not affect significantly either the sample texture or the local orientations [16]. Moreover, general agreement with standard crystal plasticity predictions on average grain rotations [19], orientation fragmentation [20] and subgrain evolution [11] supported the validity of the present split sample experiment at both the grain and subgrain scales. The grains can therefore be considered to deform as grains in a conventional, single-pieced sample. This makes microtexture tracking an attractive alternative to high-energy X-ray diffraction techniques [25, 26], especially at large strains.

\subsection{Simulation}

The crystal plasticity finite element method has been used assuming that the grains deform by slip along specific crystallographic systems. The method allows grains to develop heterogeneous strain and stress fields to match mechanical equilibrium. Details on the method and the simulation are provided in the following.

\subsubsection{Constitutive equations}

Crystal deformation is described by an elasto-viscoplastic constitutive model [22]. The model combines anisotropic elasticity and plastic deformation accommodated by slip on a restricted number of crystallographic systems. The crystal kinematics is described by a multiplicative decomposition of the deformation gradient, $\mathbf{F}$,

$$
\mathbf{F}=\mathbf{V}^{*} \mathbf{R}^{*} \mathbf{F}^{p}
$$

where $\mathbf{F}^{p}$ develops solely from slip, $\mathbf{R}^{*}$ is the lattice rotation and $\mathbf{V}^{*}$ is the elastic stretch. The decomposition defines several configurations: the initial, undeformed configuration, $\mathscr{B}_{0}$, the current, fully-deformed configuration, $\mathscr{B}$, as well as two intermediate configurations, $\mathscr{\mathscr { B }}$ and $\hat{\mathscr{B}}$, as depicted in Ref. [22]. The intermediate configurations are characterized by the presence of only some of the portions of the deformation gradient (Equation 1). Configuration $\hat{\mathscr{B}}$ is obtained from $\mathscr{B}$ by elastic unloading without rotation. Configuration $\mathscr{\mathscr { B }}$ differs from $\hat{\mathscr{B}}$ by a rotation alone. $\hat{\mathscr{B}}$ is the configuration in which the crystal constitutive behaviour is written (in the following, ' denotes the expression of ' $\bullet$ ' in $\hat{\mathscr{B}}$ ).

In most metals and metallic alloys and especially in the present case of high-temperature deformation, the elastic deformation is smaller, by several orders of magnitude, than the plastic deformation. Using small strain assumption, $\mathbf{V}^{*}$ can be approximated as,

$$
\mathbf{V}^{*}=\mathbf{I}+\varepsilon^{e}
$$

where $\mathbf{I}$ is the second-rank identity tensor and $\varepsilon^{e}$ is the infinitesimal strain tensor. The latter is related to the Kirchhoff stress by Hooke's law,

$$
\tau=\mathbb{C}: \varepsilon^{e}
$$

where $\mathbb{C}$ is the fourth-rank elasticity tensor defined in the crystal coordinate system. The Kirchhoff stress is related to the Cauchy stress, $\sigma$, by

$$
\tau=\underset{4}{\operatorname{det}}\left(\mathbf{I}+\varepsilon^{e}\right) \sigma
$$


The plastic velocity gradient, $\hat{\mathbf{L}}^{p}$, is defined from the slip-induced portion of the deformation gradient $\left(\hat{\mathbf{F}}^{p}\right)$ as

$$
\hat{\mathbf{L}}^{p}=\dot{\hat{\mathbf{F}}}^{p} \hat{\mathbf{F}}^{p^{-1}}
$$

and can be decomposed into its symmetric and skew-symmetric parts, $\hat{\mathbf{D}}^{p}$ and $\hat{\mathbf{W}}^{p}$, respectively,

$$
\hat{\mathbf{L}}^{p}=\hat{\mathbf{D}}^{p}+\hat{\mathbf{W}}^{p}
$$

$\hat{\mathbf{D}}^{p}$ and $\hat{\mathbf{W}}^{p}$ can, in turn, be related to the slip rates on the potential slip systems. Consistent with experimental observations of Pérocheau and Driver [27] on similar aluminium alloys deformed in the same conditions, a mixed slip model is used, where the octahedral $\{111\}\langle 110\rangle$ and the non-octahedral $\{110\}\langle 110\rangle$ and $\{100\}\langle 110\rangle$ systems are potentially active (24 systems). Each system is described in $\hat{\mathscr{B}}$ by its slip plane normal, $\hat{\mathbf{m}}^{\alpha}$, and its slip direction, $\hat{\mathbf{s}}^{\alpha}$. They can be combined into the Schmid tensor as $\hat{\mathbf{S}}^{\alpha} \otimes \hat{\mathbf{m}}^{\alpha}$, where $\otimes$ is the dyadic product between two vectors. The Schmid tensor can be decomposed into symmetric and skew-symmetric parts, $\hat{\mathbf{P}}^{\alpha}$ and $\hat{\mathbf{Q}}^{\alpha}$, respectively. The crystal deformation kinetics is related to crystallographic slip by

$$
\hat{\mathbf{D}}^{p}=\sum_{\alpha} \dot{\gamma}^{\alpha} \hat{\mathbf{P}}^{\alpha}
$$

and

$$
\hat{\mathbf{W}}^{p}=\dot{\mathbf{R}}^{*} \mathbf{R}^{* T}+\sum_{\alpha} \dot{\gamma}^{\alpha} \hat{\mathbf{Q}}^{\alpha}
$$

where $\dot{\gamma}^{\alpha}$ is the slip rate of system $\alpha$. Slip is assumed to follow a viscoplastic behaviour, where the slip rate $\dot{\gamma}^{\alpha}$ is related to the resolved shear stress, $\tau^{\alpha}$, through a power law [28],

$$
\dot{\gamma}^{\alpha}=\dot{\gamma}_{0}\left|\frac{\tau^{\alpha}}{g^{\alpha}}\right|^{\frac{1}{m}} \operatorname{sgn}\left(\tau^{\alpha}\right)
$$

The resolved shear stress $\tau^{\alpha}$ is given by projection of the Kirchhoff stress on system $\alpha$,

$$
\tau^{\alpha}=\hat{\mathbf{P}}^{\alpha}: \tau
$$

The slip system hardness evolution is described by

$$
\dot{g}^{\alpha}=h_{0}\left(\frac{g_{s}-g^{\alpha}}{g_{s}-g_{0}}\right) \dot{\gamma}, \quad \text { where } \dot{\gamma}=\sum_{\alpha}\left|\dot{\gamma}^{\alpha}\right|
$$

The material parameters have been identified from experimental data and are provided in Table 1 .

The crystal behaviour is used in a finite element formulation which solves for the motion of the polycrystal based on the weak form of the equilibrium equations. Given the anisotropic behaviour of the crystal, this leads to heterogeneous strain and stress fields both among and within individual grains. An extensive description of the finite element formulation can be found in Ref. [22]. Details on the simulation carried out in the present work are provided in the following. 


\subsubsection{Polycrystal description and deformation}

The grain structure was represented by a 3D 1000-grain Voronoi tessellation. 176 central grains were assigned the orientations measured on the undeformed sample. Consistent with the initial experimental texture, the surrounding grains were assigned orientations taken randomly from a uniform distribution. The exact experimental grain morphology could not be reproduced since it is known only on the observation surface. This is actually not a limitation in the context of the present study where experiment and simulation results are compared in terms of distribution over all grains, or correlation to orientation, rather than on a grain-by-grain basis. The polycrystal was discretized into equiaxed 10-node tetrahedral elements with an element size of about $30 \mu \mathrm{m}$ and then deformed according to the experimental sequence. At the end of each deformation step, the polycrystal was remeshed to eliminate element distortions resulting from strain heterogeneities. During remeshing, a constant element size $(30 \mu \mathrm{m})$ was used along RD and TD while a constant element density was retained along ND. The microstructure data were remapped from the old mesh to the new mesh (orientation and hardening parameters, $g^{\alpha}$ ) [21]. The mesh density of the central grains was of about 600 elements / grain on average at $\varepsilon=0$ and 1,250 elements / grain on average at $\varepsilon=1.2$. The entire mesh contained 258,000 elements at $\varepsilon=0$ and 446,000 elements at $\varepsilon=1.2$. Orientation maps of the microstructures obtained at successive strain levels can be found in Ref. [20]. It was shown in Ref. [21] that remeshing allows the simulation to reach large strains while retaining the grain orientation distribution yet avoiding the excessive element distortions that can terminate a simulation. All algorithms are implemented in the free (opensource) software package Neper [21, 29] (polycrystal generation, meshing and remeshing), and FEpX [22, 30] (finite element resolution of polycrystal deformation).

\section{Mathematical description of orientation and disorientation distributions}

The broadening of an orientation distribution over successively larger strains is evident from pole figures or disorientation maps, as illustrated for one grain in Figure 1. As pointed out earlier, such a "unimodal" lattice rotation, involving an average rotation and a distribution about the average orientation, is typical of $80-90 \%$ of the grains. In both experiment and simulation, a lattice orientation distribution is defined by a set of discrete orientations, $\left\{\left(\mathbf{r}^{\alpha}, \phi^{\alpha}\right)\right\}$, where $\mathbf{r}^{\alpha}$ and $\phi^{\alpha}$ are the $\alpha$ orientation and its volume fraction (area fraction in experiment), respectively. The full data sets from both experiment and simulation also include the spatial location of the local orientations (see Figure 1), but for the development here none of the spatial information is retained. Of course, an orientation distribution results from the short-distance (subgrains) and long-distance (grain-scale) orientation changes, but these gradients are disregarded to focus on the properties of the orientation distributions such as their angular extent or anisotropy (preferential disorientation axis).

Each unimodal orientation set was decomposed into an average orientation and a set of disorientations. The disorientations were computed between orientations within the original set and their average, rather than being between pixels or between elements (for experiment and simulation, respectively). We look first at the metrics of the disorientation sets for individual grains and then consider correlations between metrics across the grains. Before discussing the results, we present a summary of the methodologies for computing the average orientations and for describ- 
ing the angular extent and anisotropy of the disorientation sets. The required computations were carried out using the free (open-source) software package Orilib [31].

\subsection{Average orientation}

The average orientation can be computed efficiently by averaging quaternions [32, 33, 34, 9]. In the absence of crystal symmetry, averaging simply consists of summing all quaternions and normalizing the result to unit length. In the presence of crystal symmetry, a crystal orientation can be described by several equivalent, but distinct quaternions 11 Using any one of the symmetric equivalents arbitrarily for each orientation would lead to non-equivalent results, and so direct averaging would be erroneous [35, 34, 9]. Therefore, correct averaging requires all orientations to be expressed in the fundamental region of orientation space. Even in this case and especially for large orientations sets, as an orientation set may intersect the boundary of the fundamental region and so be divided into several distinct populations - the so-called Umklapp effect [36, 9], direct averaging would lead to an orientation far from any of the distinct populations and therefore be erroneous. This can be prevented by rotating the orientation set close to the origin of the fundamental region before averaging, i.e. working with the disorientations with respect to a reference orientation, $\mathbf{q}_{\text {ref }}$, which is close to the average orientation [34, 9]. With the orientation expressed as a quaternion written as $\mathbf{q}^{\alpha}$, the expression of an average orientation, $\overline{\mathbf{q}}$, becomes

$$
\overline{\mathbf{q}}=\left(\sum_{\alpha=1}^{N} \phi^{\alpha} \mathbf{q}_{\mathbf{d}}\left(\mathbf{q}_{\text {ref }}, \mathbf{q}^{\alpha}\right)\right) \mathbf{q}_{\mathbf{r e f}} /\left|\left(\sum_{\alpha=1}^{N} \phi^{\alpha} \mathbf{q}_{\mathbf{d}}\left(\mathbf{q}_{\text {ref }}, \mathbf{q}^{\alpha}\right)\right)\right|
$$

where $\mathbf{q}_{\mathbf{d}}\left(\mathbf{q}_{\text {ref }}, \mathbf{q}^{\alpha}\right)$ is the disorientation between $\mathbf{q}_{\text {ref }}$ and $\mathbf{q}^{\alpha}$ expressed in the reference coordinate system and ' $|\bullet|$ ' is the norm of ' $\bullet$ '. $\mathbf{q}_{\mathbf{d}}\left(\mathbf{q}_{\text {ref }}, \mathbf{q}^{\alpha}\right)$ can be written as

$$
\mathbf{q}_{\mathbf{d}}\left(\mathbf{q}_{\mathbf{r e f}}, \mathbf{q}^{\alpha}\right)=\min _{i=1}^{24}\left[\left(\mathbf{q}^{\alpha} \mathbf{u}_{\mathbf{i}}\right) \mathbf{q}_{\mathbf{r e f}}{ }^{-1}\right]
$$

where $\mathbf{u}_{\mathbf{i}}$ stands for the $i$ th cubic symmetry operator and 'min' designates the minimum in terms of rotation angle. For small orientation sets, $\mathbf{q}_{\text {ref }}$ can be chosen as an arbitrary orientation of the set. In contrast, particularly large orientation sets may still be the object of the Umklapp effect depending on the initial value of $\mathbf{q}_{\text {ref }}$. In such a case, an iterative procedure is required to refine the value of $\mathbf{q}_{\text {ref }}$ and therefore $\overline{\mathbf{q}}$, where at each iteration, $\mathbf{q}_{\text {ref }}$ is set to the previously computed $\overline{\mathbf{q}}$ value. The average orientation expressed as a Rodrigues vector is designated as $\overline{\mathbf{r}}$. The disorientation between $\mathbf{r}^{\alpha}$ and $\overline{\mathbf{r}}$, expressed in the reference coordinate system, is designated as $\mathbf{w}^{\alpha}$.

\subsection{Angular extent and anisotropy}

The angular extent of a disorientation set (thereby of an orientation distribution) can be simply characterized by the average disorientation angle,

$$
\bar{\theta}=2 \sum_{i=1}^{N} \phi^{\alpha} \arctan \left|\mathbf{w}^{\alpha}\right|
$$

\footnotetext{
${ }^{1}$ Of course, this is also true for other descriptors: rotation matrices, Euler angles, Rodrigues vectors, etc.
} 
The anisotropy of a disorientation set can be described by metrics proposed by Glez and Driver [9] and Barton and Dawson [37]. Similar formulations have been subsequently provided by Pantleon [38] and Bachmann et al. [39]. A $3 \times 3$ covariant matrix, $\mathbf{S}$, is built on the disorientation set

$$
\mathbf{S}=\sum_{i=1}^{N} \phi^{\alpha}\left(\mathbf{w}^{\alpha} \otimes \mathbf{w}^{\alpha}\right)
$$

which is symmetric and can be diagonalized..$^{2}$ The eigenvectors, $\mathbf{v}_{\mathbf{i}}(i \in\{1,2,3\})$, provide the principal axes of the distribution and the eigenvalues, $\lambda_{i}\left(\lambda_{1} \geq \lambda_{2} \geq \lambda_{3}\right)$, provide the characteristic lengths along the axes. The preferential direction of the disorientation set is provided by the first eigenvector $\left(\mathbf{v}_{1}\right)$, associated with the highest eigenvalue $\left(\lambda_{1}\right)$. This is the rotation axis about which the disorientation angles are the highest and will be referred to as "preferential disorientation axis" in the following. The angular components of the disorientation vectors along the principal axes can be obtained as

$$
\theta_{i}^{\alpha}=2 \arctan \left(\mathbf{v}_{\mathbf{i}} \cdot \mathbf{w}^{\alpha}\right)
$$

As an example, the disorientation distribution and anisotropy properties corresponding to the grain of Figure 1 at $\varepsilon=1.2$ are provided in Figure 2. They are unimodal and typical of orientation distributions produced by plastic deformation [10]. The strength of the anisotropy can be characterized by an "anisotropy factor", $\lambda_{a}$, defined by

$$
\lambda_{a}=\lambda_{1} / \sqrt[3]{\lambda_{1} \lambda_{2} \lambda_{3}}
$$

which takes the minimum value of 1 for an isotropic disorientation set.

\section{Evolution of intra-grain disorientations during plane strain compression}

The intra-grain orientation distributions were derived for the monitored grains at each of the successive strain levels for both the experiment and simulation. Metrics of the distributions were computed for those experimental grains for which there were at least 1000 orientation measurements at each strain level, which corresponds to a minimum apparent grain size of $180 \mu \mathrm{m}$. A total of 92 grains of the complete set of 176 monitored grains met this requirement. The rejected grains were either grains with an effective diameter larger than $180 \mu \mathrm{m}$, but with an apparent diameter (on the observation surface) less than $180 \mu \mathrm{m}$, or grains with an effective diameter less than $180 \mu \mathrm{m}$. In principle, rejecting the smallest grains may somewhat bias the results, but the size criterion was necessary to ensure that the intra-grain disorientation metrics were representative of the sample atlarge. The same set of 92 grains was considered in the simulation metrics, with a slight difference due to a limited number of grains that underwent orientation fragmentation in the experiment, but not in the simulation, or vice versa. In the following, we first analyse trends in the metrics of the disorientation distributions over this set of grains. We then look for correlations between the metrics of the disorientation distributions and the grain average orientations.

\footnotetext{
${ }^{2}$ The original expression of Ref. [9] includes a quadratic term which in practice is negligible and is omitted here.
} 


\subsection{Average disorientation angle and anisotropy factor}

The distributions of the average disorientation angles are presented in Figure 3 for the experiment and simulation. Average values as successive strains are listed in Table 2 , For both experiment and simulation, the average disorientation angle increases rapidly early in the deformation $(\varepsilon<0.5)$ to comparable values between 5 and $6^{\circ}$. At strains beyond 0.5 , the rate of increase drops off more rapidly in the experiment than in the simulation, with the experimental average tending towards a saturation of $7-8^{\circ}$. Although the rate of increase is diminishing in the simulation, it is difficult to estimate what the saturation value, if any, might be. In both simulation and experiment there is a high degree of variability in the average disorientation, ranging in the experiment from 3.6 to $19.7^{\circ}$.

The distributions of the anisotropy factors are presented in Figure 4 for experiment and simulation. The average values are given in Table 2 along with the average disorientation angles. The trends from experiment and simulation show less similarity than we observed for the average disorientation angle. While in both experiment and simulation the values of $\lambda_{a}$ are between 1 and 2 , in the experiment values decrease with strain while in the simulation they increase. A possible explanation for these opposite trends will be provided in Section 6

\subsection{Frequency distribution of the preferential disorientation axes}

The distributions of preferential disorientation axes at successive strains are provided on Figure 5, as equal-area projections onto the sample RD-ND plane, for both experiment and simulation. The projections are reduced to one quarter using orthotropic sample symmetry. In addition, the simulated distribution at $\varepsilon=0.02$ is also provided to analyse the early stage of plastic deformation. Figure 5 shows that the experimental axes are strongly aligned with TD up to $\varepsilon=0.42$. Further, at higher strains, some of the axes tend to rotate toward other directions. At $\varepsilon=1.2$, the distribution is fairly equally balanced between the original TD and RD, but also contains axes located halfway between RD and ND. The latter direction will be referred to as 'RDND' in the following. In the simulation, the axes also are close to TD at small strains. A transition then occurs at lower strains than in experiment, between $\varepsilon=0.19$ and 0.42 , and the large-strain distribution is shared between RD and ND, without any TD. It is often found that simulated textures develop faster than the experimental ones [19, 40, 41] and this appears to be also true for the disorientation axes.

In principle, the preferential disorientation axes can be correlated with directions other than sample directions. One possibility is a correlation with a crystal lattice direction. This can be analysed by expressing the preferential disorientation axes in the crystal coordinate system and plotting them in the crystal stereographic triangle. The axes appeared nearly uniformly distributed, especially at small strains, meaning no correlation. A second possibility is a correlation with the grain average rotation direction: as the latter is also distributed about TD [16], we may ask whether the preferential disorientation axis of a grain is more strongly correlated with TD or with its average rotation axis. For each grain, the correlations can be quantified from the angles between the preferential disorientation axis and either TD or the average rotation axis. For most grains, a significantly lower angle was found with TD, which confirms a stronger correlation of the preferential disorientation axis with TD. 


\subsection{Correlation of the preferential disorientation axes with grain average orientations}

To investigate the relationships between the preferential disorientation axes and the corresponding grain average orientations, the preferential axes can be plotted in the fundamental region of the Rodrigues orientation space. As extensively described by Kumar and Dawson [42], Rodrigues space has many advantages over the more commonly used Euler space, at least for reorientation analysis of cubic symmetry (here face-centered cubic) materials. First, the cubic Rodrigues fundamental region has the shape of a 3D polyhedron (a truncated cube). Second, orientation fibres appear as straight lines and the $\alpha$ and $\beta$ texture fibres are located at the surface of the space, as shown on Figure 6, where the locations of the ideal components are also indicated. Finally, the fundamental region shows a relatively low distortion without degeneracy.

The distributions of the preferential axes in the fundamental region, at successive strains and for both experiment and simulation, are plotted in Figure 7. A preferential disorientation axis is represented as a small baton as follows:

- The radius and length are constant.

- The barycentre is on the average orientation.

- The axis is along the preferential disorientation axis, in orientation space $3^{3}$

- The colour is related to the preferential disorientation axis components. Axes aligned with $\mathrm{RD}$, TD or ND are coloured red, green or blue, respectively. Intermediate positions are represented by mixed colours, as indicated on Figure 7 .

Finally, orthotropic sample symmetry is applied, so that each preferential disorientation axis is actually represented by 4 batons.

It can be clearly seen from the distributions that, at small strains, the batons are nearly uniformly distributed in orientation space, which is due to the weak initial texture, and mostly aligned with TD, which is consistent with previous results (Figure 5, $\varepsilon=0.02-0.42$ ). As the strain increases, the batons continuously migrate toward the $\alpha$ and $\beta$ fibres and rotate. At $\varepsilon=1.2$, most batons have converged upon the fibres. In the experiment, the preferential disorientation axes are shared between TD, RD and RDND. In the simulation, they are shared between RD and ND; only one grain has retained a TD preferential disorientation axis. Again, this is consistent with Figure 5. In the simulation, a preferential disorientation axis clearly aligns with the direction of the fibre to which the associated average orientation belongs. The same trend can be found in the experimental distribution by careful visual inspection (for axes which do not still align with TD). It also appears that for the experiment, the area of ND-oriented preferential disorientation axes extends up to the Goss orientation while in the simulation, it is restricted to the vicinity of the $\beta$ fibre. This explains the difference between the experimental and simulated distributions of the preferential disorientation axes that can be seen on Figure 5 .

\footnotetext{
${ }^{3}$ Because the composition of 2 rotations, $\mathbf{r}_{\mathbf{1}}$ and $\mathbf{r}_{\mathbf{2}}$, in Rodrigues space is given by $\left(\mathbf{r}_{1}, \mathbf{r}_{\mathbf{2}}\right)=$ $\left(\mathbf{r}_{1}+\mathbf{r}_{2}-\mathbf{r}_{1} \times \mathbf{r}_{2}\right) /\left(1-\mathbf{r}_{1} \cdot \mathbf{r}_{2}\right)$ (when both orientations are expressed in the same, reference coordinate system), i.e. is not the parallelogram law for vectors, in the general case, rotations about the same axis, but from 2 different orientations, will result in different translations in Rodrigues space. For example, a rotation about ND from the Cube orientation corresponds to a shift along the $(0,0,1)$ direction while the same rotation from the Goss orientation corresponds to a shift along $\sim(0,-0.38,0.92)$. The advantage of taking this difference into account in the present graphical representation is that a baton aligns with the associated orientation distribution.
} 


\section{Relation to deformation mechanisms}

The development of an intra-grain orientation distribution with straining indicates that different lattice rotations have occurred over the grain volume. Differences in lattice rotation result from differences in slip system activity, which are driven by the local stress. We can therefore expect that, at the early state of plastic deformation, the development of the grain orientation distribution depends on both the crystal slip geometry and the stress heterogeneities. Further, at larger strains, sub-volumes within grains can possess such distinct lattice orientations that significantly different reorientations will result under continued deformation. This section analyses the implications of these phenomena on the intra-grain orientation distributions, and in particular their preferential disorientation axes. In Section 5.1, the anisotropy of the intra-grain orientation distributions at low strain is related to the slip system activity using the simulation results. We then construct a simplified model based on local variations of the stresses predicted using a Taylor assumption to identify the fundamental mechanisms leading to the preferential disorientation axes (Section 5.2). At high strain, we turn again to the Taylor assumption as a starting point and consider how variations in the initial lattice orientation can give rise to orientation distributions with preferential disorientation axes under the action of large strain deformations (Section 5.3). In both instances, the Taylor assumption provides an estimate of spin about which fluctuations arising from spatial heterogeneities of stress and deformation are constructed by different means.

\subsection{Slip geometry and variability}

To accommodate an arbitrary plastic deformation regardless of its lattice orientation, a crystal must possess 5 independent slip systems. However, it is commonly the case that fewer, just 2 or 3 , dominate the slip activity during plastic straining. The slip geometry can be analysed by looking at the distributions of the slip plane normals $\mathbf{m}^{\alpha}$, slip directions $\mathbf{s}^{\alpha}$ and spin directions $\mathbf{t}^{\alpha}=\mathbf{m}^{\alpha} \times \mathbf{s}^{\alpha}$ for the activated systems, which are available in the simulation results. Instead of limiting the distributions to the 2 or 3 most activated systems, all systems are taken into account and their vectors are weighted by the associated average absolute slip rates $\left(\left|\bar{\gamma}^{\alpha}\right|\right)$. The distributions over all grains of the slip vectors at $\varepsilon=0.02$ are provided in Figure 8 . Both the $\mathbf{m}^{\alpha}$ and $\mathbf{s}^{\alpha}$ vectors show distributions about ND tilted by $45^{\circ}$ about TD (also called 'RDND'). The corresponding spin vectors $\mathbf{t}^{\alpha}$ are preferentially distributed about TD. This actually corresponds to the systems of maximum Schmid factor for plane strain compression. It should be noted that such distributions are a consequence of the deformation mode imposed on the grain, and to first order, are independent of either the grain average orientation or the level of strain. Similar distributions would be provided by simplified models such as the Taylor or self-consistent models.

The slip rate variability within a grain can also be extracted from the simulation results. This is first carried out at $\varepsilon=0.02$, with the advantage that all elements are close to the initial orientation and therefore have slip systems oriented approximately in the same way. For each grain, the slip rate variability on a specific system can be quantified by the standard deviation of the slip rates over all elements. In Figure 9, the slip rate variabilities of all systems of all grains are plotted against the corresponding average slip rates. It can be seen that the systems of high average slip rates exhibit the highest slip rate variabilities, while nonactive systems exhibit low variabilities. The higher slip rate variabilities on the most active slip systems, combined with their spin vectors close to 
$\mathrm{TD}$, lead to higher reorientation rates about TD and in the end to a preferential disorientation axis distribution about TD, as seen in Figure 5 for $\varepsilon=0.02-0.42$. Conversely, equivalent slip rate variabilities on all slip systems would lead to equivalent reorientation velocity variabilities in all directions and therefore to no preferential disorientation axes. A deeper understanding requires, however, to analysing the influence of a stress distribution on the reorientation distribution inside a grain.

\subsection{Influence of stress variability}

As with the description of the orientation distributions, the stress heterogeneities inside the grains are cast as frequency distributions without regard to the spatial dependence. The influence of stress variability on reorientations can be evaluated quantitatively from the crystal plasticity theory described in Section 2.2.1 (Equations 4, 8, 9, and 10p. Here, the nominal stress can be evaluated using the Taylor assumption of equal plastic strain, zero elastic strain and zero spin, i.e. $\mathbf{W}^{\mathbf{p}}=\mathbf{0}, \varepsilon^{e}=\mathbf{0}$ and therefore $\hat{\bullet}=\bullet$. Since we are interested in the development of the orientation distributions from the undeformed state, $\mathbf{R}^{*}=\mathbf{I}$. By rewriting Equation 8 , it follows that the reorientation velocity $\left(\dot{\mathbf{R}}^{*}\right)$ is equal to the opposite of the plastic spin,

$$
\dot{\mathbf{R}}^{*}=-\sum_{\alpha} \dot{\gamma}^{\alpha} \mathbf{Q}^{\alpha}
$$

or equivalently, in vectorial form,

$$
\dot{\mathbf{r}}^{*}=-\sum_{\alpha} \dot{\gamma}^{\alpha} \mathbf{t}^{\alpha}
$$

where $\dot{\mathbf{r}}^{*}$ is the reorientation velocity vector. The slip rates $\dot{\gamma}^{\alpha}$ are related to the resolved shear stresses $\tau^{\alpha}$ by Equation 9 , and, under the zero elastic strain assumption, the resolved shear stresses are related to the Cauchy stress by (Equations 4 and 10 )

$$
\tau^{\alpha}=\mathbf{P}^{\alpha}: \sigma
$$

where both $\mathbf{P}^{\alpha}$ and $\sigma$ are expressed in the sample reference system.

In the context of a rate-dependent material behaviour (Equation 9), the slip rate on a system is non-zero if the resolved shear stress is non-zero. A change of stress is therefore expected to affect the slip rates of all systems and ultimately the reorientation velocity (Equation 19).

Let $\sigma_{v}=\left(\sigma_{11}, \sigma_{22}, \sigma_{33}, \sqrt{2} \sigma_{23}, \sqrt{2} \sigma_{31}, \sqrt{2} \sigma_{12}\right)$ denote the stress in vectorial form. The variation of reorientation velocity as a function of stress can be evaluated from the 2 nd-rank, $3 \times 6$ tensor $\frac{\partial \dot{\mathbf{r}}^{*}}{\partial \sigma_{v}}$, as

$$
\frac{\partial \dot{\mathbf{r}}^{*}}{\partial \sigma_{v}}=\frac{\partial}{\partial \sigma_{v}}\left(-\sum_{\alpha} \dot{\gamma}^{\alpha} \mathbf{t}^{\alpha}\right)
$$

Because $\mathbf{t}^{\alpha}$ does not depend on $\sigma_{v}$ and by elementary mathematical manipulations, we have

$$
\frac{\partial \dot{\mathbf{r}}^{*}}{\partial \sigma_{v}}=-\sum_{\alpha}\left(\mathbf{t}^{\alpha} \otimes \frac{\partial \dot{\gamma}^{\alpha}}{\partial \sigma_{v}}\right)
$$


As $\dot{\gamma}^{\alpha}$ and $\sigma_{v}$ are related via the resolved shear stresses $\tau^{\alpha}$, the expression can appropriately be rewritten as,

$$
\frac{\partial \dot{\mathbf{r}}^{*}}{\partial \sigma_{v}}=-\sum_{\alpha}\left(\mathbf{t}^{\alpha} \otimes \frac{\partial \dot{\gamma}^{\alpha}}{\partial \tau^{\alpha}} \frac{\partial \tau^{\alpha}}{\partial \sigma_{v}}\right)
$$

The first partial derivative is known from the kinetics expression given by Equation 9 ,

$$
\frac{\partial \dot{\gamma}^{\alpha}}{\partial \tau^{\alpha}}=\frac{\dot{\gamma}_{0}}{m g^{\alpha}}\left|\frac{\tau^{\alpha}}{g^{\alpha}}\right|^{\frac{1}{m}-1}
$$

or, in terms of slip rate $\dot{\gamma}^{\alpha}$,

$$
\frac{\partial \dot{\gamma}^{\alpha}}{\partial \tau^{\alpha}}=\frac{\dot{\gamma}_{0}}{m g^{\alpha}}\left|\frac{\dot{\gamma}^{\alpha}}{\dot{\gamma}_{0}}\right|^{1-m}
$$

For rate sensitivities typical of cold or hot-deformed metals (here, $m=0.12$ ), $\frac{\partial \dot{\gamma}^{\alpha}}{\partial \tau^{\alpha}}$ is a strongly decreasing function of $\tau^{\alpha}$ and a nearly linear function of $\dot{\gamma}^{\alpha}$.

The second partial derivative in the expression is known from the Schmid law together with the assumption of no elastic strain (Equation 20),

$$
\frac{\partial \dot{\mathbf{r}}^{*}}{\partial \sigma_{v}}=-\sum_{\alpha}\left(\mathbf{t}^{\alpha} \otimes \frac{\partial \dot{\gamma}^{\alpha}}{\partial \tau^{\alpha}} \frac{\partial\left(\mathbf{p}^{\alpha} \cdot \sigma_{v}\right)}{\partial \sigma_{v}}\right)
$$

where $\mathbf{p}^{\alpha}$ stands for the vector form of the symmetrical Schmid tensor $\mathbf{P}^{\alpha}, \mathbf{p}^{\alpha}=\left(P_{11}^{\alpha}, P_{22}^{\alpha}, P_{33}^{\alpha}\right.$, $\left.\sqrt{2} P_{23}^{\alpha}, \sqrt{2} P_{31}^{\alpha}, \sqrt{2} P_{12}^{\alpha}\right)$. After inserting these relations and making some minor rearrangement, one obtains

$$
\frac{\partial \dot{\mathbf{r}}^{*}}{\partial \sigma_{v}}=-\sum_{\alpha} \frac{\partial \dot{\gamma}^{\alpha}}{\partial \tau^{\alpha}}\left(\mathbf{t}^{\alpha} \otimes \mathbf{p}^{\alpha}\right)
$$

The factor $\left(\mathbf{t}^{\alpha} \otimes \mathbf{p}^{\alpha}\right)$ is fully geometrical and depends only on the crystal lattice orientation.

The expression of $\frac{\partial \dot{\mathbf{r}}^{*}}{\partial \sigma_{v}}$ provides the variation of the reorientation velocity vector as a function of a variation of the stress vector. The question then is: if a grain does not show a unique stress state, but instead a random, isotropic stress distribution about the nominal value, does it develop a particular, anisotropic reorientation velocity distribution? This can be ascertained from the singular value decomposition of $\frac{\partial \dot{\mathbf{r}}^{*}}{\partial \sigma_{v}}$,

$$
\frac{\partial \dot{\mathbf{r}}^{*}}{\partial \sigma_{v}}=\mathbf{U S} \mathbf{V}^{T}
$$

where $\mathbf{U}$ is a $(3,3)$ orthogonal tensor, $\mathbf{S}$ is a $(3,6)$ diagonal tensor and $\mathbf{V}$ is a $(6,6)$ orthogonal tensor. The diagonal entries of $\mathbf{S}$ are sorted in decreasing order. It is important to note that $\mathbf{U}$ is a basis of the physical (real) space and $\mathbf{V}$ is a basis of the stress space. A random, isotropic stress distribution (random vectors in $\mathbf{V}$ ) leads to a reorientation velocity distribution of principal directions given by the rows of $\mathbf{U}$ and magnitudes given by the associated diagonal entries of $\mathbf{S} 4^{4}$

\footnotetext{
${ }^{4}$ A prerequisite is to write the stress vector with $\sqrt{2}$ factors for the shear components, so that a random, isotropic distribution in stress space leads to equal distribution magnitudes along the different components.
} 
The reorientation velocity distributions described by the $\mathbf{U}$ and $\mathbf{S}$ tensors can be compared to the experimental and simulated small-strain disorientation distributions in terms of anisotropy factor and preferential axis. The distribution of the anisotropy factors, $S_{a}=S_{11} / \sqrt[3]{S_{11} S_{22} S_{33}}$, over all initial orientations is provided in Figure 10. It ranges from 1 (no anisotropy) to values as high as 6, with an average value of 3, which is somewhat higher than observed in the experiment and simulation (average values of 1.9 and 1.6, respectively; see Figure 4). This can be considered similar to the tendency of the Taylor model to overestimate the rate of texture development. The distribution of the associated preferential directions are provided in Figure $11 \mathrm{a}$, together with the correlation to orientation in Figure $11 \mathrm{p}$ and the distribution over the Rodrigues fundamental region in Figure 11 c,d. The distribution exhibits an intense focus about TD that is in remarkable quantitative agreement with observations (Figures 5 and 7 at $\varepsilon=0.02-0.19$ ). The distribution over the Rodrigues fundamental region confirms that a TD preferential disorientation axis is expected for almost all orientations. Only rare orientations (the exact Cube orientation and orientations close to U) are predicted to develop non-TD preferential disorientation axes.

The general trend to develop TD preferential disorientation axes can be explained from the expression of $\frac{\partial \dot{\mathbf{r}}^{*}}{\partial \sigma_{v}}$ (Equation 27), which involves the slip geometry and the slip behaviour. Under the effect of a random, isotropic stress distribution about the nominal value, all slip systems are subjected to equivalent resolved shear stress distributions. On a given slip system, the resolved

shear stress perturbations lead to slip rate perturbations proportional to $\frac{\partial \dot{\gamma}}{\partial \tau}$. The $\frac{\partial \dot{\gamma}}{\partial \tau}$ values are significantly higher for the active systems, which have high $\tau^{\alpha}$ (or equivalently, $\dot{\gamma}^{\alpha}$ ) values and spin vectors close to TD (Section 5.1 and Figure 8). This leads to higher slip rate variabilities along the most active systems, as can be seen in Figure 9, and ultimately to a reorientation velocity perturbation which has a higher component along TD. To an isotropic stress distribution will therefore correspond a reorientation velocity distribution preferentially aligned with TD.

\subsection{Influence of reorientation velocity field conditions}

The characteristics of the reorientation velocity field also plays a key role on orientation distributions. The Taylor model reorientation velocity field for plane strain compression, which depends only on orientation, is provided in Figure 12 on the surface of the Rodrigues fundamental region. This reorientation velocity field shows basic features associated with texture development. The orientations rotate quickly toward one of the stable fibres then rotate more slowly along the fibre towards a stable orientation. This reproduces well both experimental and simulated average rotations (see Section 4.3). The influence of the reorientation velocity conditions on an orientation distribution can first be interpreted from the velocity divergence, as provided in Figure 13a [42, 43]. A negative value indicates a flow converging on an orientation, while a positive value indicates a divergent flow. Not surprisingly, the $\alpha$ and $\beta$ fibres exhibit very low (negative) divergence values associated with regions exhibiting a strongly convergent velocity field (Figure 12). Under negative divergence, the spread of an orientation distribution narrows, while positive divergence causes it to broaden. In general, the reorientation velocity conditions around a particular orientation are different along different directions. This can be characterized from the eigen decomposition of the velocity gradient. It should be noted that real (non-complex) eigenvalues are obtained only if the velocity gradient is symmetrical. This is actually not strictly the case, but the skew-symmetric part 
has been found to be significantly smaller than the symmetric part, by at least one order of magnitude, and zero or nearly zero in the vicinity of the $\alpha$ and $\beta$ fibres. Therefore, for the development here, only the symmetric part of the velocity gradient is taken into account. If an eigenvalue is negative, the flow is convergent along the associated principal direction. If it is positive, the flow is divergent. Under the effect of different eigenvalues, a spherical (isotropic) orientation distribution would therefore become ellipsoidal, or equivalently, develop a preferential disorientation axis. Recalling that $\operatorname{div}\left(\dot{\mathbf{r}}^{*}\right)=\sum_{i=1}^{3} \lambda_{i}$ and $\lambda_{1}=\max _{i=1}^{3} \lambda_{i}$, the velocity gradient anisotropy, $\mu$, can be quantified as

$$
\mu=\lambda_{1}-\frac{1}{3} \operatorname{div}\left(\dot{\mathbf{r}}^{*}\right)
$$

which takes the minimal value of 0 for isotropic divergence. The principal direction associated with $\lambda_{1}$ constitutes the preferential disorientation axis. The velocity gradient anisotropy and preferential disorientation axis distributions are provided in Figures $13 \mathrm{~b}$ and $13 \mathrm{k}$, respectively. The velocity gradient anisotropy changes considerably over orientation space, as does the associated preferential disorientation axis. However, the stable fibres are surrounded by wide, well-defined preferential axis regions associated with high velocity gradient anisotropies. The $\beta$ fibre exhibits regions of RD and RDND axes while the $\alpha$ fibre exhibits regions of RD and ND axes (note the wide ND-axis region about the Goss orientation). The distribution of the preferential axes for the experimental and simulated final orientations $(\varepsilon=1.2)$ are provided in Figures $14 \mathrm{a}, \mathrm{b}$ (to be compared with Figures $5 \mathrm{a}, \mathrm{b}$ at $\varepsilon=1.2$ ). Both Taylor distributions are similar, as expected from the similar experimental and simulated grain orientation distributions. The distributions show axes extending from RD to ND in 3 distinct populations: RD (density 5), RDND (density 8) and ND (density 2 in experiment only). The distributions are in qualitative agreement with the components arising through strain in the observed distributions. The principal discrepancies relate to the balance between the RD and RDND populations in the experiment, and on the presence of a population extending from RD to RDND and a large population about ND in the simulation. This can be further analysed from the correlation of the preferential disorientation axes to the average orientations as illustrated on Figure 14k,d (to be compared with Figure 7k). Again, both distributions, built from the experimental and simulated final orientations, exhibit similar trends. The region about the $\beta$ fibre up to Goss exhibits either RD or RDND axes, while a region around Goss toward Cube exhibits ND axes. This is also in qualitative agreement with the observed distributions. Differences are on some TD axes left in the experiment, and an ND area extending from Goss to Copper in the simulation. 


\section{Discussion}

For both experiment and simulation, the orientation distributions were found to grow rapidly up to $\varepsilon=0.5$. In this regime, the preferential disorientation axes were shown to distribute about TD, in agreement with previous results on single crystals [10] and grains of a cold-rolled polycrystal [38]. Compared to the cold-deformation results of Pantleon and coauthors [38], the preferential disorientation axes are more intensely focused about TD, by a factor of 2 on the distribution density. This can be attributed to the activation of the non-octahedral slip systems in the present hightemperature deformation, which favors slip on systems aligned with planes of maximum shear (ideally at $45^{\circ}$ between ND and RD) and having spin vectors parallel to TD. This in turn leads to preferential disorientation axes about TD, driven in part by random spatial variations in the stress.

At strains beyond 0.5 , the average disorientation angle tends to approach a saturation limit of $7-8^{\circ}$ in experiment, consistent with previous experimental results on single crystals [10]. The rate of increase in the simulation decreases with strain, but remains higher than observed in the experiment. In the experiment, the preferential disorientation axes shift continuously from TD to RD or RDND. The simulation produced a similar trend, except that the simulation evolves more quickly and also includes a shift toward ND. This is important information, especially in the industrial context where most metallic alloys are hot-rolled to strains of 1-1.5. At large strains, an orientation dependency of the preferential disorientation axes has also been found, especially in the simulation, from RD around the Brass orientation to RDND around the Copper and S orientations and to ND around the Goss orientation.

The development of preferential disorientation axes has been related to two different mechanisms:

- Slip geometry and stress variability. In deformed single crystals and grains of a polycrystal, it is well known that stress is not homogeneous. Within individual crystals, dislocations interact and annihilate (at high temperature) to form cell structures associated with short-range stress heterogeneities. Across polycrystals, the interactions of anisotropic grains results in complex, long-range stress heterogeneities. The stress heterogeneities induce spatial fluctuations in the deformation and variability in the local slip system activity associated with plastic deformation. Intra-grain orientation distributions arise out of the variability in reorientation velocity inherent with spatially-varying slip. The influence of the stress variability on the reorientation velocity variability can be quantified through the derivative of the reorientation velocity vector with respect to the stress vector, $\frac{\partial \dot{\mathbf{r}}^{*}}{\partial \sigma_{v}}$. This quantity is written as

$$
\frac{\partial \dot{\mathbf{r}}^{*}}{\partial \sigma_{v}}=-\sum_{\alpha} \frac{\partial \dot{\gamma}^{\alpha}}{\partial \tau^{\alpha}}\left(\mathbf{t}^{\alpha} \otimes \mathbf{p}^{\alpha}\right)
$$

and depends both on the slip geometry (the slip systems combined with the grain orientation) and the slip behaviour. A singular value decomposition was used to determine the strength of the reorientation velocity anisotropy caused by a random, isotropic, intra-grain stress distribution. The resulting anisotropies (and therefore the orientation distribution anisotropy) were found to be high (average value of 3) and the preferential axes were found to be about TD. This indicates that the preferential disorientation axis distribution about TD is 
caused by the tendency of the crystal to develop a distribution of reorientation velocities (about a nominal value) preferably about TD even for a random, isotropic stress distribution. This is due to the fact that the highest slip variabilities are found on the most active systems, which exhibit spin vectors preferably distributed about TD. Such an influence of slip conditions on the most active slip systems has also been observed recently by Oddershede and coauthors [44] for a specific lattice orientation in a Copper polycrystal deformed in tension. The expression of $\frac{\partial \mathbf{r}^{*}}{\partial \sigma_{v}}$ also indicates that the lower the temperature (i.e. the rate sensitivity, $m$ ), the stronger the orientation distribution anisotropy. Of course, in a real material, the stress distribution is not expected to be isotropic, but the present agreement for the TD disorientation axes at small strains indicates that any potential anisotropy of the stress distributions can only have a second order effect.

- Reorientation velocity conditions. When an orientation distribution is sufficiently broad, its orientations can be subject to different reorientations even under the same stress or strain. This influences the orientation distribution anisotropy and can be analysed from the reorientation velocity field. The eigen decomposition of the velocity gradient indicates how an orientation distribution narrows or broadens about specific directions during deformation. The directions (and the associated intensities) change widely over orientation space, but the values of greatest interest are those in the vinicity of stable fibres. The predicted directions evolve smoothly along the $\beta$ and $\alpha$ fibres from RD about the Brass orientation, to RDND about the $\mathrm{S}$ and Copper orientations, and to ND about the Goss orientation.

These two mechanisms favor different disorientation axes and compete to provide an evolution of the preferential disorientation axis distributions with strain that is dominated at low strain by one mechanism and large strain by the other. Assuming a constant stress variability in the grains, the stress-variability mechanism predicts a linear growth of the average disorientation angle with stable, intense anisotropy (anisotropy factor of 3 on average) and preferential disorientation axis distribution about TD. In contrast, the reorientation-velocity mechanism influences the anisotropy properties only when the orientation distributions are large enough and most grains have reached the stable texture fibres. By continuously broadening the orientation distributions more along a direction between RD and ND (the first eigenvector of the reorientation velocity gradient, which depends on the orientation) than the original TD, this second mechanism leads to a transition to RD or RDND. The finite element simulation intrinsically combines both mechanisms to provide a continuous evolution of the orientation distributions throughout straining which is in qualitative agreement with experiment results. The transition of preferential disorientation axes is accompanied by an evolution of the anisotropy factors. In the experiment, anisotropy factors decrease. In the simulation, however, anisotropy factors increase, which can be related to the faster preferential disorientation axis transition, effective at a strain level of about 0.5. Once the orientation distribution of a grain has aligned with the direction provided by the reorientation velocity conditions, it will continue to broaden more along this direction than others, leading to an increase of the anisotropy factor. This is what clearly happens in the simulation at strains beyond 0.5 . This is not seen in the experiment because the axis transition is not fully effective even at the final strain of 1.2. The present mechanism indicates, however, that further deformation would cause all the 
preferential disorientation axes to migrate toward either RD or RDND and the anisotropy factors to increase.

Interestingly, the transition of the preferential disorientation axes from TD to RD or RDND also indicates a change of the dislocation structures, i.e. the character of the dislocation subgrains. Given that the slip system spin vectors are preferably distributed about TD throughout the straining, orientation of the disorientation axes provides insight regarding the character of the dislocations. Specifically, a TD preferential disorientation axis at small strain indicates a higher content of edge dislocations (Burgers vector perpendicular to the rotation axis) while an RD or RDND preferential disorientation axis at large strain indicates a higher content of screw dislocations (Burgers vector parallel to the rotation axis).

\section{Conclusions}

A quantitative analysis of the evolution of orientation distributions in a hot, plane-strain compressed Al-0.1 wt.\% Mn polycrystal has been carried out by a combined experiment-simulation approach. The microtexture tracking experiment provides the orientations and reorientations of 92 grains with more than 1000 measurements per grain. In the simulation, each grain was discretized into several hundreds of elements, also giving access to intra-grain orientation distributions. The results were analysed in terms of average disorientation angle and anisotropy, and in particular preferential disorientation axes. The average disorientation angles were found to increase rapidly and then to saturate at $\varepsilon=0.5$ at about $7^{\circ}$ in average. The anisotropy intensity was found to decrease with strain. The preferential disorientation axes were found to be distributed about TD up to $\varepsilon=0.5$ and then to migrate to RD or RDND, depending on the grain average orientation. These two attributes of the microstructure evolution are influenced by two competing mechanisms: (i) isotropic stress variations about the nominal stress that lead to strong anisotropy of the orientation distribution with preferential disorientation axes aligned with TD and (ii) reorientation velocity gradients that tend to spread the orientation distribution along RD, RDND, or ND in the vicinities of the stable $\beta$ and $\alpha$ fibres.

\section{Acknowledgments}

Partial support has been provided by ONIR under grant number NOON14-09-1-0447 


\section{References}

[1] N. Hansen, New discoveries in deformed metals, Metall. Mater. Trans. A 32A (2001) 2917-2935.

[2] F. Humphreys, Modelling mechanisms and microstructures of recrystallisation, Mater. Sci. Tech. 8 (1992) 135143.

[3] D. Hughes, N. Hansen, Microstructure and strength of Nickel at large strains, Acta Mater. 48 (2000) $2985-3004$.

[4] Q. Liu, X. Huang, D. Lloyd, N. Hansen, Microstructure and strength of commercial purity aluminium (AA 1200) cold-rolled to large strains, Acta Mater. 50 (2002) 3789-3802.

[5] G. Pennock, M. Drury, C. Spiers, The development of subgrain misorientations with strain in dry synthetic $\mathrm{NaCl}$ measured using EBSD, Journal of Structural Geology 27 (2005) 2159-2170.

[6] H. McQueen, J. Hockett, Microstructures of aluminum compressed at various rates and temperatures, Metall. Trans. 1 (1970) 2997-3004.

[7] K. Marthinsen, E. Nes, Modelling strain hardening and steady state deformation of Al-Mg alloys, Mater. Sci and Techn. 17 (2001) 376-388.

[8] F. Humphreys, P. Bate, The microstructures of polycrystalline Al0.1Mg after hot plane strain compression, Acta Mater. 55 (2007) 5630-5645.

[9] J.-C. Glez, J. Driver, Orientation distribution analysis in deformed grains, J. Appl. Crystallogr. 34 (2001) 280288.

[10] J.-C. Glez, J. Driver, Substructure development in hot plane strain compressed Al-1\%Mn crystals, Acta Mater. 51 (10) (2003) 2989-3003.

[11] R. Quey, J. Driver, Microtexture tracking of sub-boundary evolution during hot deformation of aluminium, Mater. Charac. 62 (2011) 1222-1227.

[12] W. Pantleon, W. He, T. Johansson, C. Gundlach, Orientation Inhomogeneities within Individual Grains in Coldrolled Aluminium resolved by Electron Backscatter Diffraction, Mater. Sc. Eng., A 483-484 (2008) 668-671.

[13] W. He, W. Ma, W. Pantleon, Microstructure of individual grains in cold-rolled aluminium from orientation inhomogeneities resolved by electron backscattering diffraction, Mater. Sc. Eng., A 494 (2008) 21-27.

[14] S. Krog Petersen, J. Bowen, W. Pantleon, Quantitative characterization of the orientation spread within individual grains in copper after tensile deformation, Int J Materials Research 100 (2009) 433-438.

[15] L. Tóth, C. Gu, Modelling of disorientation axis distribution in severely deformed copper, Scr. Mater. 69 (2013) 183-186.

[16] R. Quey, D. Piot, J. Driver, Microtexture tracking in hot-deformed polycrystalline aluminium: Experimental results, Acta Mater. 58 (2010) 1629-1642.

[17] C. Barrett, Structure of iron after compression., Trans A.I.M.E. 135 (1939) 296-324.

[18] F. Humphreys, M. Hatherley, Recrystallization and Related Annealing Phenomena, Springer, 1996.

[19] R. Quey, D. Piot, J. Driver, Microtexture tracking in hot-deformed polycrystalline aluminium: Comparison with simulations, Acta Mater. 58 (2010) 2271-2281.

[20] R. Quey, P. Dawson, J. Driver, Grain orientation fragmentation in hot-deformed aluminium: Experiment and simulation, J. Mech. Phys. Solids 60 (2012) 509-524.

[21] R. Quey, P. R. Dawson, F. Barbe, Large-scale 3D random polycrystals for the finite element method: Generation, meshing and remeshing, Comput. Methods Appl. Mech. Engrg. 200 (2011) 1729-1745.

[22] E. B. Marin, P. R. Dawson, On modelling the elasto-viscoplastic response of metals using polycrystal plasticity, Comput. Methods Appl. Mech. Engrg. 165 (1998) 1-21.

[23] C. Barrett, L. Levenson, The Structure of Aluminum after Compression, Trans A.I.M.E. 137 (1939) 112.

[24] S. Panchanadeeswaran, R. Doherty, R. Becker, Direct observation of orientation change by channel die compression of polycrystalline aluminum-Use of a split sample, Acta Mater. 44 (3) (1996) 1233-1262.

[25] H. Poulsen, L. Margulies, S. Schmidt, G. Winther, Lattice rotations of individual bulk grains: Part I: 3D X-ray characterization, Acta Mater. 51 (13) (2003) 3821-3830.

[26] R. Pokharel, J. Lind, A. Kanjarla, R. Lebensohn, S. Fai Li, P. Kenesei, Suter R.M., A. Rollett, Polycrystal Plasticity: Comparison Between Grain-Scale Observations of Deformation and Simulations, Annu. Rev. Condens. Matter Phys 5 (2014) 317-346.

[27] F. Pérocheau, J. Driver, Slip system rheology of Al-1\% Mn crystals deformed by hot plane strain compression, Int. J. Plast. 18 (2) (2002) 185-202. 
[28] J. Hutchinson, Creep and plasticity of hexagonal polycrystals as related to single crystal slip, Metall Trans A 8 (1977) 1465-1469.

[29] R. Quey, Neper: a 3D random polycrystal generator for the finite element method (version 2.0), http://neper.sourceforge.net (2013).

[30] P. Dawson, D. Boyce, FEpX - Finite Element Polycrystals: Theory, Finite Element Formulation, Numerical Implementation and Illustrative Examples, ArXiv arXiv:1504.03296 [cond-mat.mtrl-sci].

[31] R. Quey, Orilib: a collection of routines for orientation manipulation, http://orilib.sourceforge.net, 2nd Edition (2008).

[32] N. Krieger Lassen, D. Jensen, K. Conradsen, On the Statistical Analysis of Orientation Data, Acta Crystall. A50 (1994) 741-748.

[33] M. Humbert, N. Gey, J. Muller, C. Esling, Determination of a Mean Orientation from a Cloud of Orientations. Application to Electron Back-Scattering Pattern Measurements, J. Appl. Crystallogr. 26 (1996) 662-666.

[34] F. Humphreys, P. Bate, P. Hurley, Orientation averaging of electron backscattered diffraction data, J. Microsc. 201 (2001) 50-58.

[35] A. Morawiec, A Note on Mean Orientation, J. Appl. Crystallogr. 31(5) (1998) 818-819.

[36] F. Frank, Orientation mapping, Metall. Mater. Trans. A 19 (3) (1988) 403-408.

[37] N. R. Barton, P. R. Dawson, On the spatial arrangement of lattice orientations in hot-rolled multiphase titanium, Modelling ans Simulation in Materials Science and Engineering 9 (2001) 433-463.

[38] W. Pantleon, Retrieving Orientation Correlations in Deformation Structures from Orientation Maps, Mater. Sci. Technol. 21 (2005) 1392-1396.

[39] F. Bachmann, R. Hielscher, P. Jupp, W. Pantleon, H. Schaeben, E. Wegert, Inferential statistics of electron backscatter diffraction data from within individual crystalline grains, J. App. Cryst. 43 (2010) 1338-1355.

[40] S. Harren, The finite deformation of rate-dependent polycrystals-II: A comparison of the self-consistent and Taylor methods, Journal of the Mechanics and Physics of Solids 39 (3) (1991) 361-383.

[41] C. Maurice, J. Driver, Hot rolling textures of f.c.c. metals-Part II. Numerical simulations, Acta Mater. 45 (11) (1997) 4639-4649.

[42] A. Kumar, P. Dawson, Dynamics of texture evolution in face-centered cubic polycrystals, J. Mech. Phys. Solids 57 (2009) 422-445.

[43] D. Raabe, Z. Zhao, W. Mao, On the dependence of in-grain subdivision and deformation texture of aluminum on grain interaction, Acta Mater. 50 (17) (2002) 4379-4394.

[44] J. Oddershede, J. Wright, Beaudoin A., G. Winther, Deformation-induced orientation spread in individual bulk grains of an interstitial-free steel, Acta Materialia 85 (2015) 301-313. 


\section{List of Figures}

1 Example of a grain undergoing unimodal rotation (grain \#9). (a) $\varepsilon=0.19$, (b) $\varepsilon=$ 0.42 , (c) $\varepsilon=0.77$ and (d) $\varepsilon=1.2$. At the top are the pole figures (stereographic projections) of the orientation distributions, where the initial orientation is represented by the dots. Below each pole figure is a map of the disorientation vector, $\mathbf{w}$, measured with respect to the average orientation of the deformed grain geometry. Components of $\mathbf{w}$ are assigned RGB colour levels using: $255 \times w_{i} / \tan \left(\theta_{\max } / 2\right)$

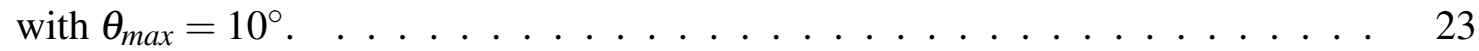

2 Anisotropy analysis of an orientation distribution, exemplified by the case of Figure 11 at $\varepsilon=1.2$. (a) Disorientation distribution in the Rodrigues fundamental region and principal directions $\left(\mathbf{v}_{\mathbf{1}}, \mathbf{v}_{\mathbf{2}}\right.$ and $\left.\mathbf{v}_{\mathbf{3}}\right)$. (b) Disorientation distributions about the 3 principal directions $\left(\theta_{1}, \theta_{2}\right.$ and $\left.\theta_{3}\right)$. The associated average disorientation angles are 5.7, 3.5 and $2.6^{\circ}$, respectively. The anisotropy factor is equal to

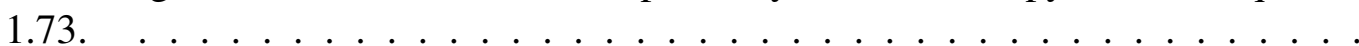

3 Evolution of the intra-grain average disorientation angles $(\bar{\theta})$ with strain in (a) experiment and (b) simulation. (In the simulation, the actual maximum value at $\varepsilon=1.2$ is $29^{\circ}$ and is out of range). Average values are provided in Table 2 . . . . .

$4 \quad$ Evolution of the intra-grain anisotropy factor $\left(\lambda_{a}\right)$ with strain in (a) experiment and (b) simulation. Average values are provided in Table 2 . . . . . . . . . 26

5 Evolution of the preferential disorientation axes with strain (equal-area projections). The preferential disorientation axes of the grains are plotted as points, with a density field and contour lines added. (a) $\varepsilon=0.02$, (b) $\varepsilon=0.19$, (c) $\varepsilon=0.42$, (d) $\varepsilon=0.77$ and (e) $\varepsilon=1.20$. Left is experiment and right is simulation. . . . . 27

6 Ideal orientations and fibres for plane strain compression of an FCC polycrystal. The coordinates of the ideal components can be found in Ref. [42]. . . . . . . . . 28

7 Correlation between the preferential disorientation axes and the average orientations of the grains, illustrated in the Rodrigues fundamental region. For each grain, the preferential disorientation axis is represented as a baton located at the average orientation of the grain. The colour of the axis is related to its direction. (a) $\varepsilon=0.02$, (b) $\varepsilon=0.19$, (c) $\varepsilon=0.42$, (d) $\varepsilon=0.77$ and (e) $\varepsilon=1.2$. Left is experiment and right is simulation. $\ldots \ldots \ldots \ldots$

8 Geometry of the active slip systems for the 92 grains in simulation, represented as the distributions of (a) $\left|\dot{\gamma}^{\alpha}\right| \overline{\mathbf{m}}^{\alpha}$, (b) $\left|\overline{\dot{\gamma}^{\alpha}}\right| \overline{\mathbf{s}^{\alpha}}$ and (c) $\left|\overline{\dot{\gamma}^{\alpha}}\right| \overline{\mathbf{t}}^{\alpha}$ (equal-area projections).

9 Relation between the slip rate variability and the average slip rate. The data are plotted for the slip systems of the 92 grains in simulation and the variability stands for the standard deviation. Each point comes from a specific slip system of a grain (24 points per grain). . . . . . . . . . . . . . . . 32

10 Intra-grain orientation distribution anisotropy factors resulting from an isotropic stress variability $\ldots \ldots \ldots \ldots \ldots \ldots \ldots \ldots \ldots \ldots \ldots \ldots \ldots$ 
11 Preferential disorientation axes resulting from an isotropic stress variability shown as (a) distribution over all initial orientations (equal-area projection), (b) correlation with the associated average orientations, (c) distribution over the Rodrigues fundamental region and (d) slices along the sample symmetry planes. $\ldots . . . . \quad 34$

12 Reorientation velocity field represented on the surface of the Rodrigues fundamental region. Note the convergence of the velocity field about the $\alpha$ and $\beta$ fibres (shown in blue and red, respectively). Compared to Figure 6, the ideal $\beta$ fibre has been bent to match the convergence line of the velocity field. . . . . . . . . . 35

13 Properties of the Taylor reorientation velocity field. (a) Divergence, (b) velocity gradient anisotropy and (c) first principal axis (corresponding to the highest prin-

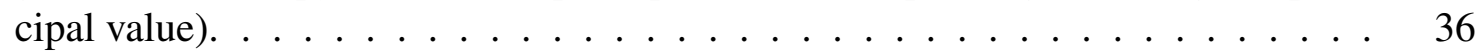

14 Preferential disorientation axes provided by the Taylor reorientation velocity field shown as distribution over all (a) experimental and (b) simulated final orientations, and correlation with the associated average orientations for (c) experiment and (d) simulation. (a) and (b) are equal-area projections. . . . . . . . . . . . 37 
$\{111\}$

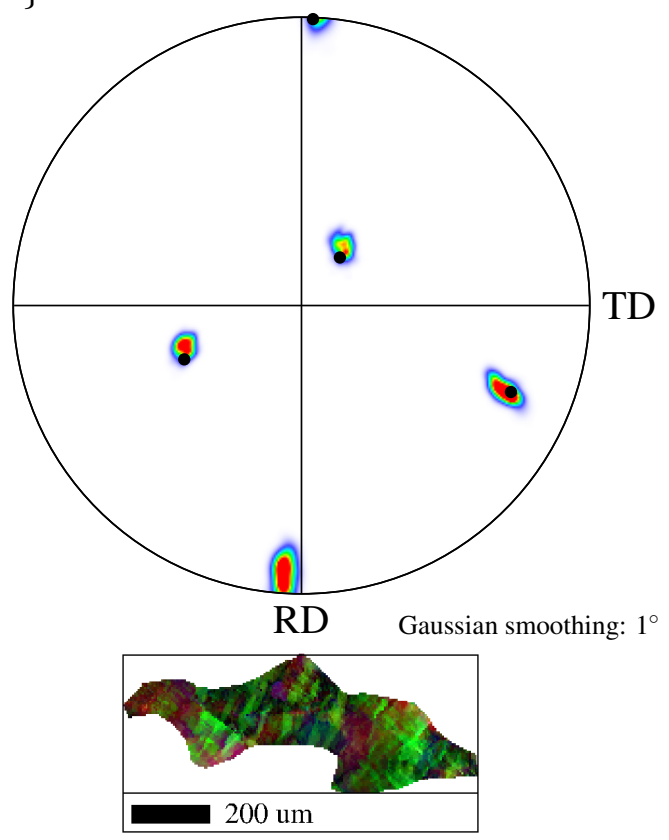

(a)

$\{111\}$

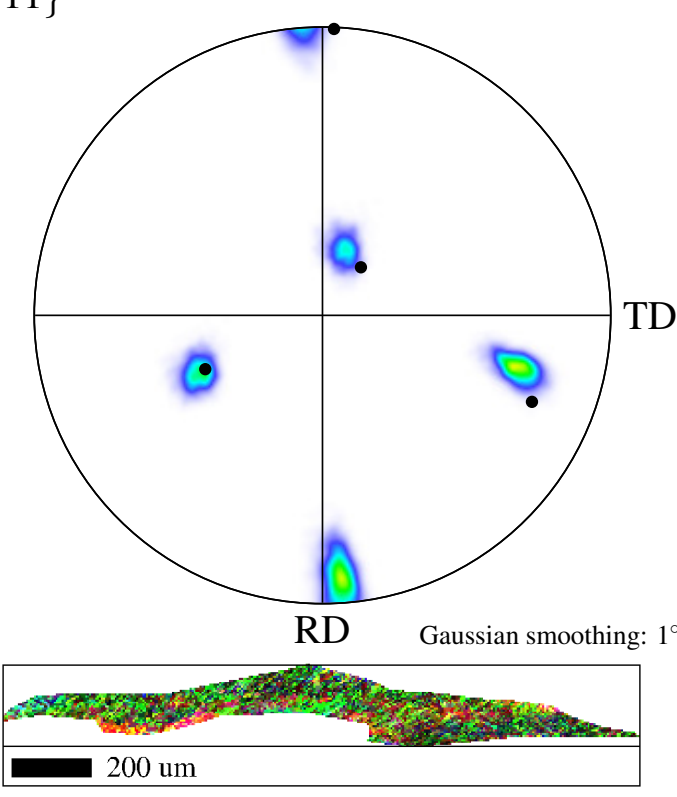

(c)
$\{111\}$

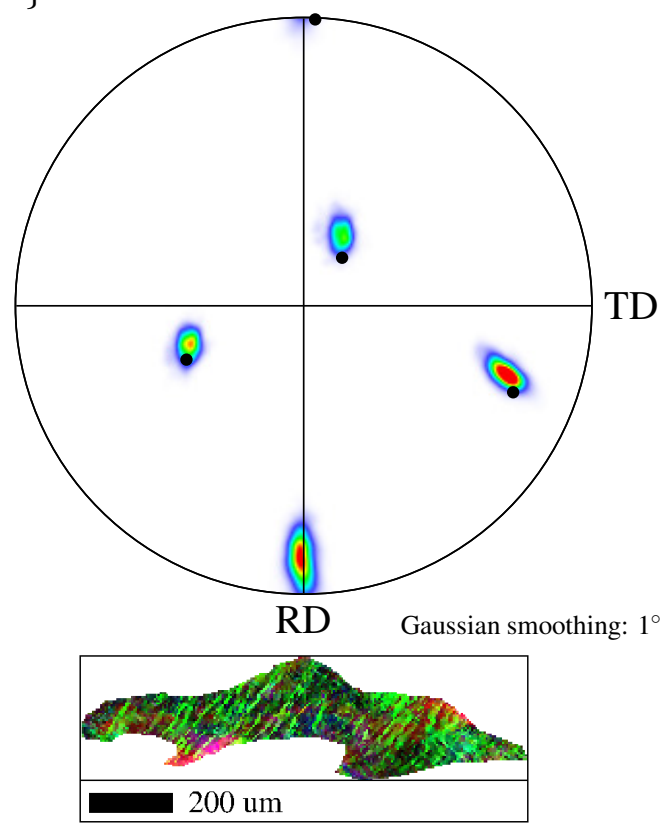

(b)

$\{111\}$

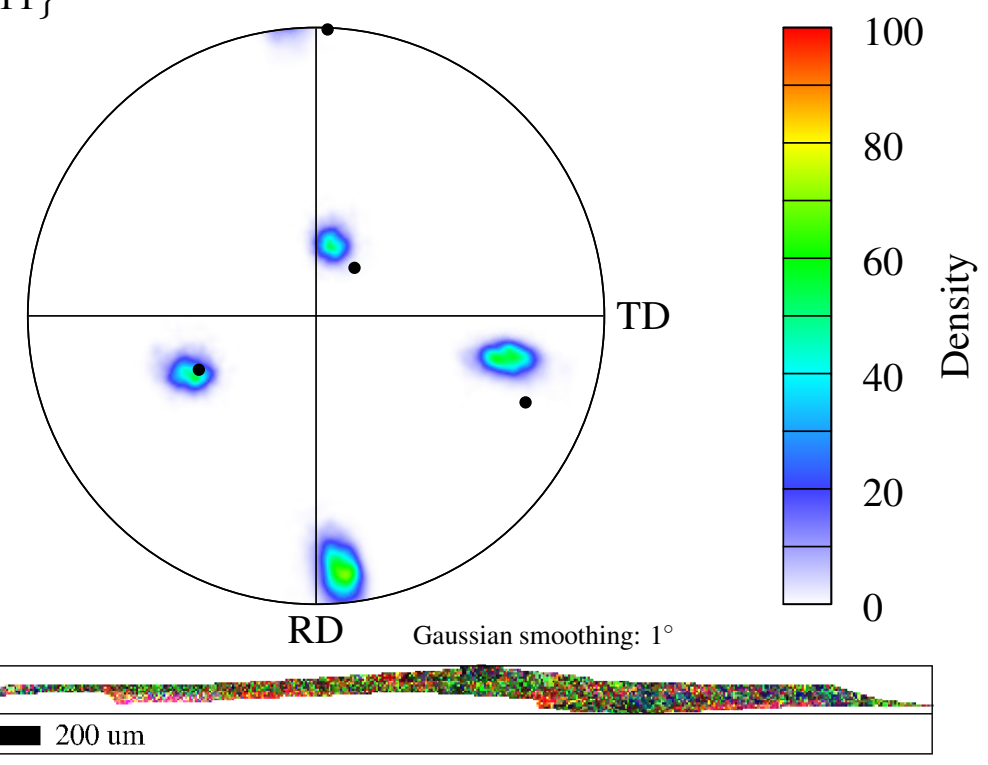

(d)

Figure 1: Example of a grain undergoing unimodal rotation (grain \#9). (a) $\varepsilon=0.19$, (b) $\varepsilon=0.42$, (c) $\varepsilon=0.77$ and (d) $\varepsilon=1.2$. At the top are the pole figures (stereographic projections) of the orientation distributions, where the initial orientation is represented by the dots. Below each pole figure is a map of the disorientation vector, $\mathbf{w}$, measured with respect to the average orientation of the deformed grain geometry. Components of $\mathbf{w}$ are assigned RGB colour levels using: $255 \times w_{i} / \tan \left(\theta_{\max } / 2\right)$ with $\theta_{\max }=10^{\circ}$. 

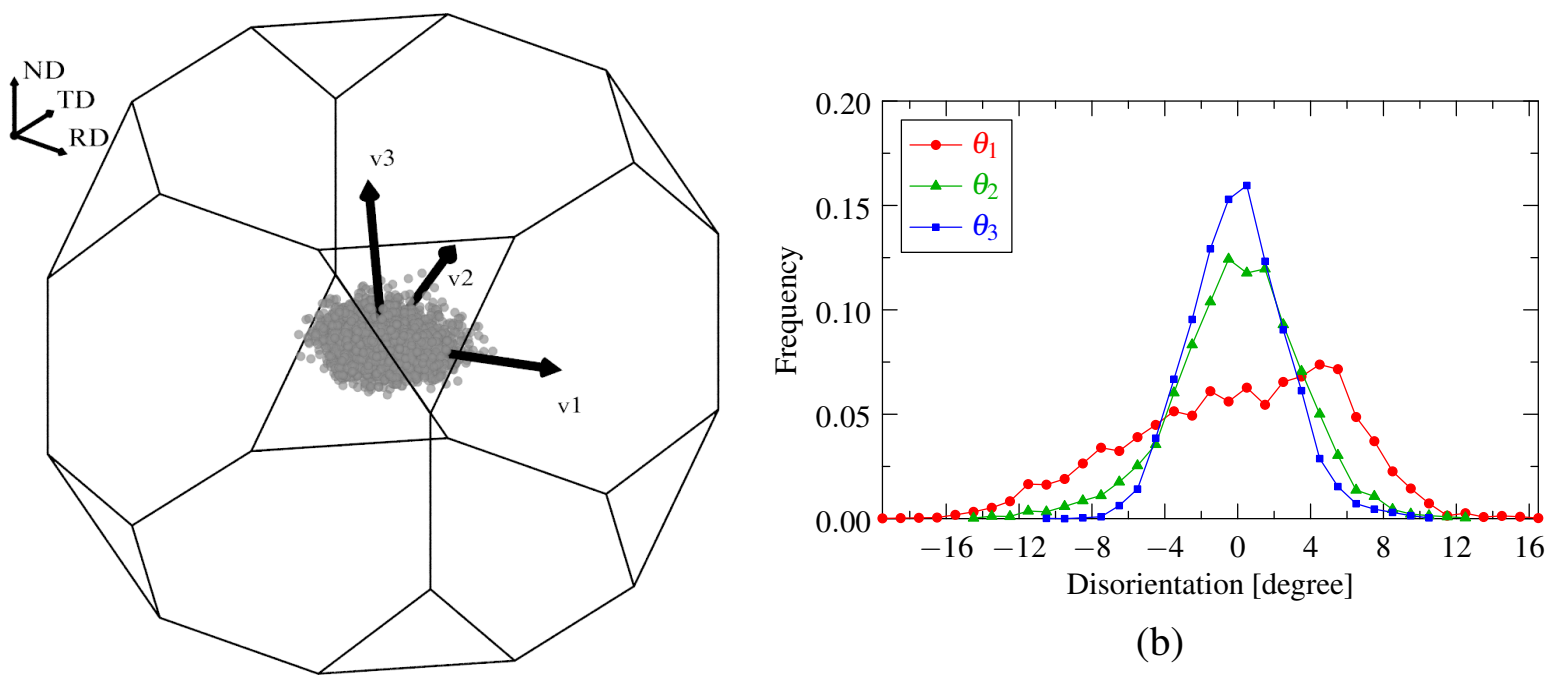

(b)

(a)

Figure 2: Anisotropy analysis of an orientation distribution, exemplified by the case of Figure 1 at $\varepsilon=1.2$. (a) Disorientation distribution in the Rodrigues fundamental region and principal directions $\left(\mathbf{v}_{\mathbf{1}}, \mathbf{v}_{\mathbf{2}}\right.$ and $\left.\mathbf{v}_{\mathbf{3}}\right)$. (b) Disorientation distributions about the 3 principal directions $\left(\theta_{1}, \theta_{2}\right.$ and $\left.\theta_{3}\right)$. The associated average disorientation angles are 5.7, 3.5 and $2.6^{\circ}$, respectively. The anisotropy factor is equal to 1.73 . 


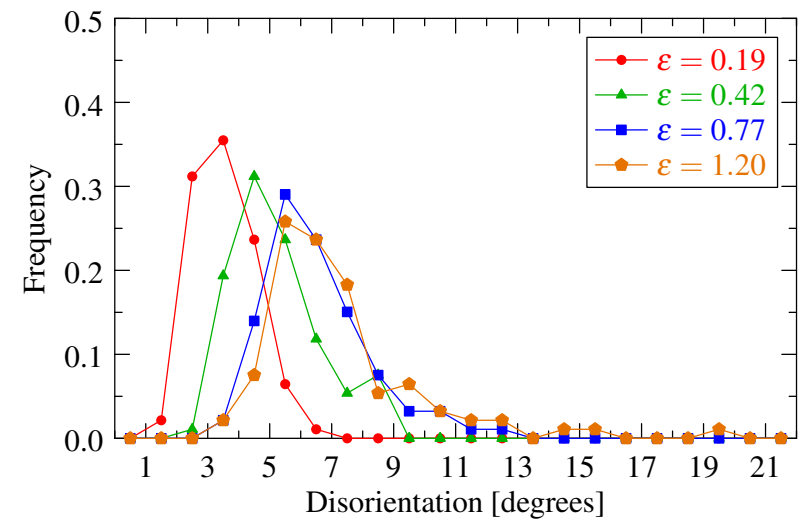

(a)

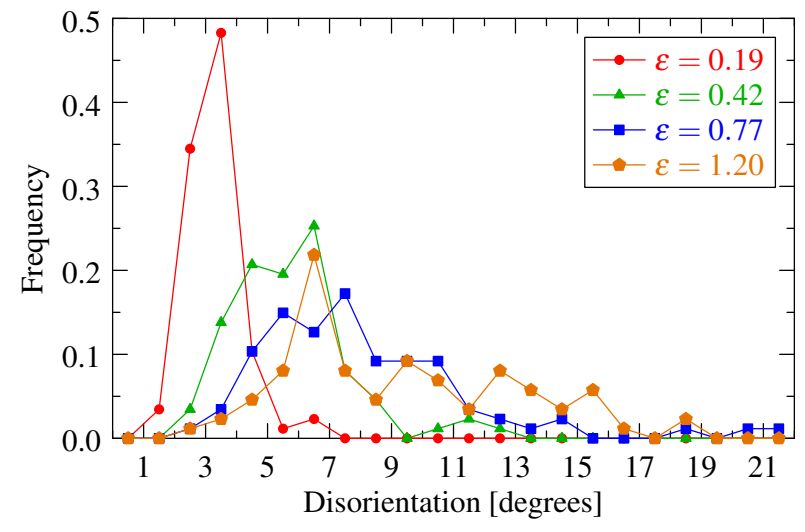

(b)

Figure 3: Evolution of the intra-grain average disorientation angles $(\bar{\theta})$ with strain in (a) experiment and (b) simulation. (In the simulation, the actual maximum value at $\varepsilon=1.2$ is $29^{\circ}$ and is out of range). Average values are provided in Table 2 


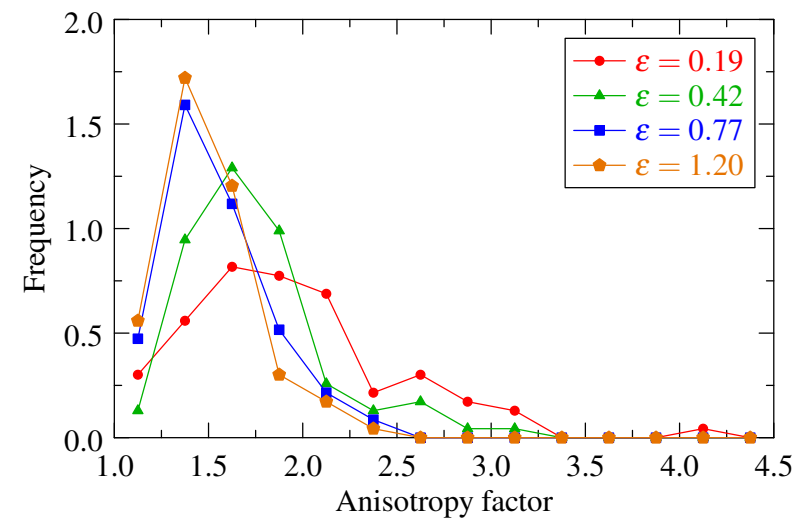

(a)

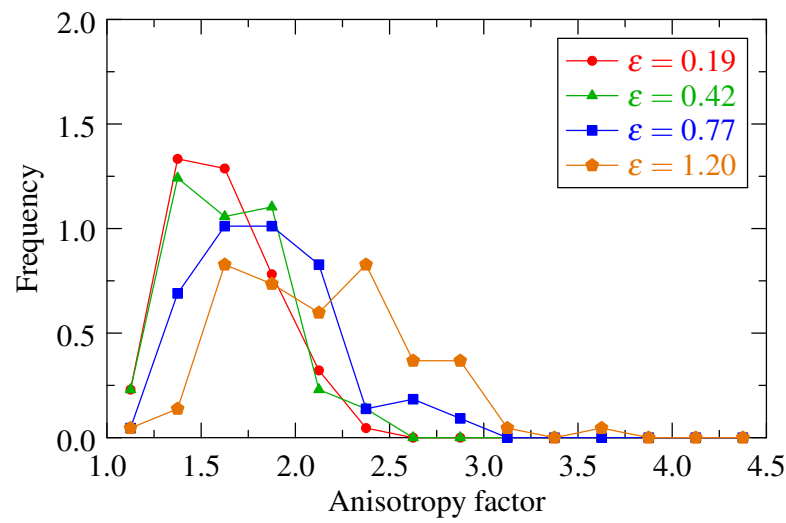

(b)

Figure 4: Evolution of the intra-grain anisotropy factor $\left(\lambda_{a}\right)$ with strain in (a) experiment and (b) simulation. Average values are provided in Table 2 
(a)

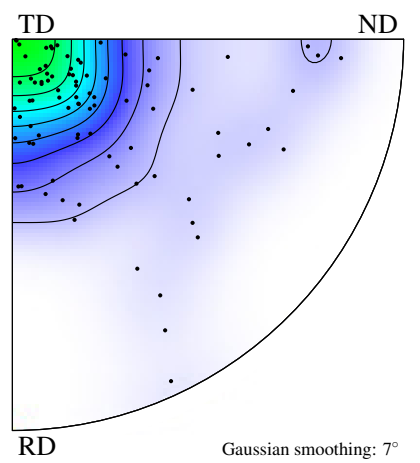

(b)
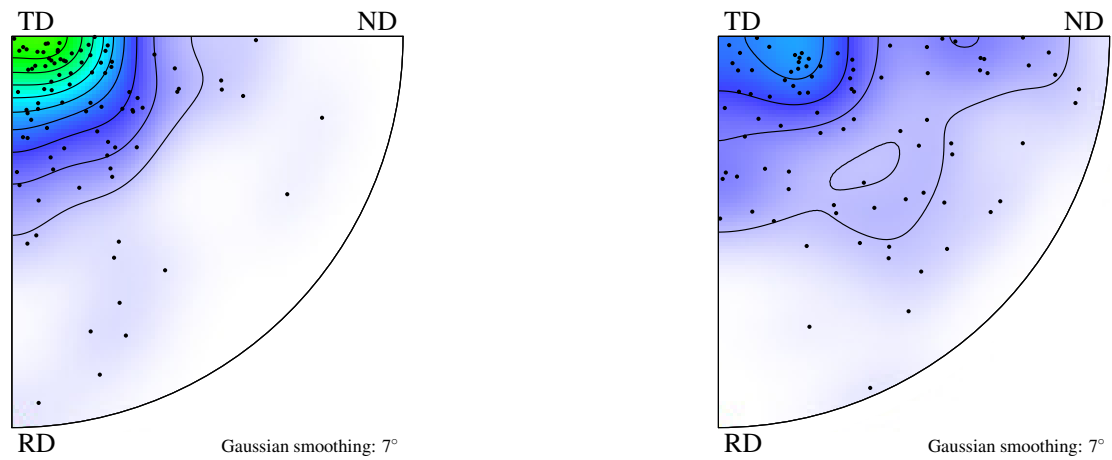

(c)
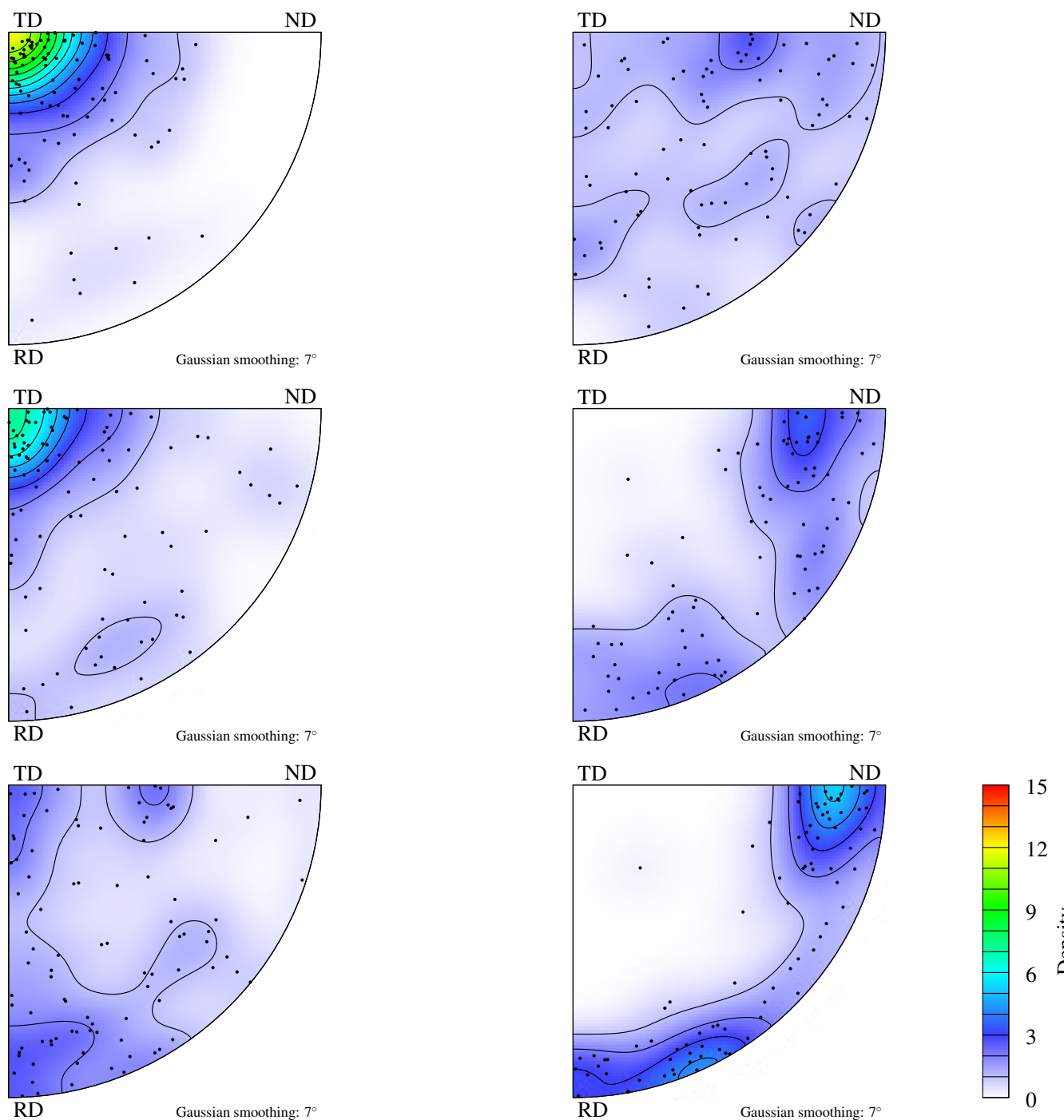

Figure 5: Evolution of the preferential disorientation axes with strain (equal-area projections). The preferential disorientation axes of the grains are plotted as points, with a density field and contour lines added. (a) $\varepsilon=0.02$, (b) $\varepsilon=0.19$, (c) $\varepsilon=0.42$, (d) $\varepsilon=0.77$ and (e) $\varepsilon=1.20$. Left is experiment and right is simulation. 


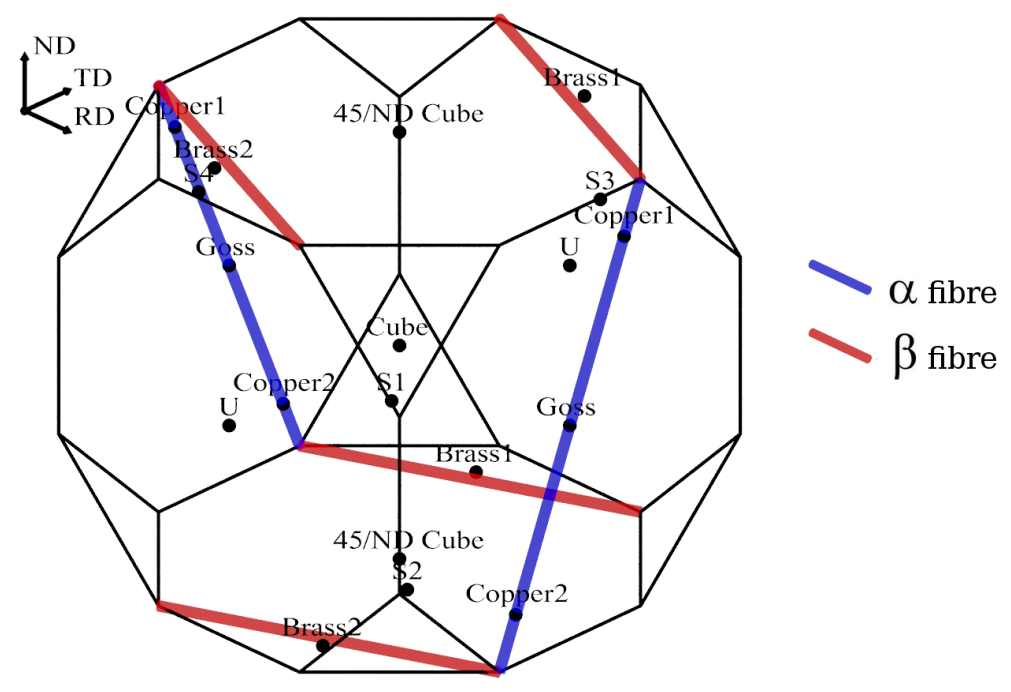

Figure 6: Ideal orientations and fibres for plane strain compression of an FCC polycrystal. The coordinates of the ideal components can be found in Ref. [42]. 
(a)

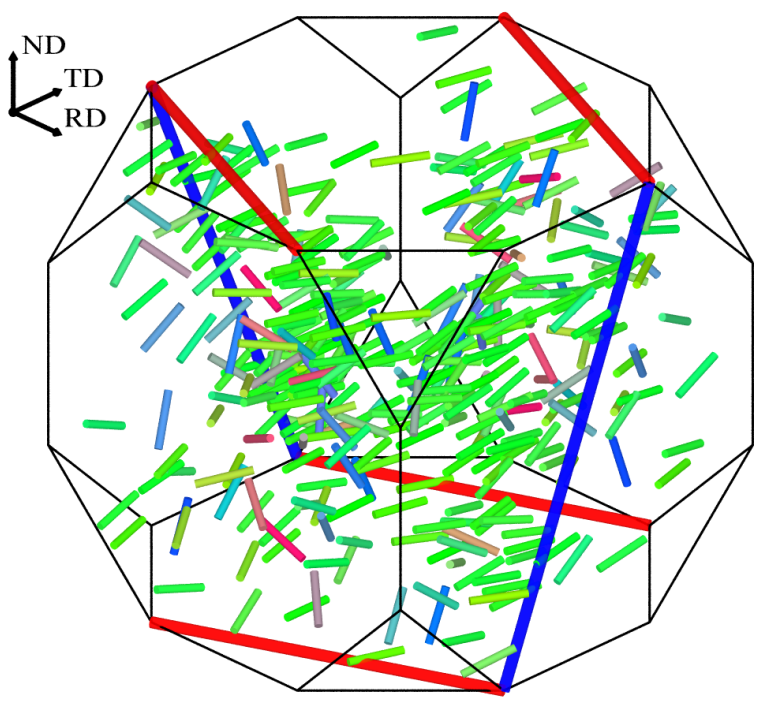

(b)
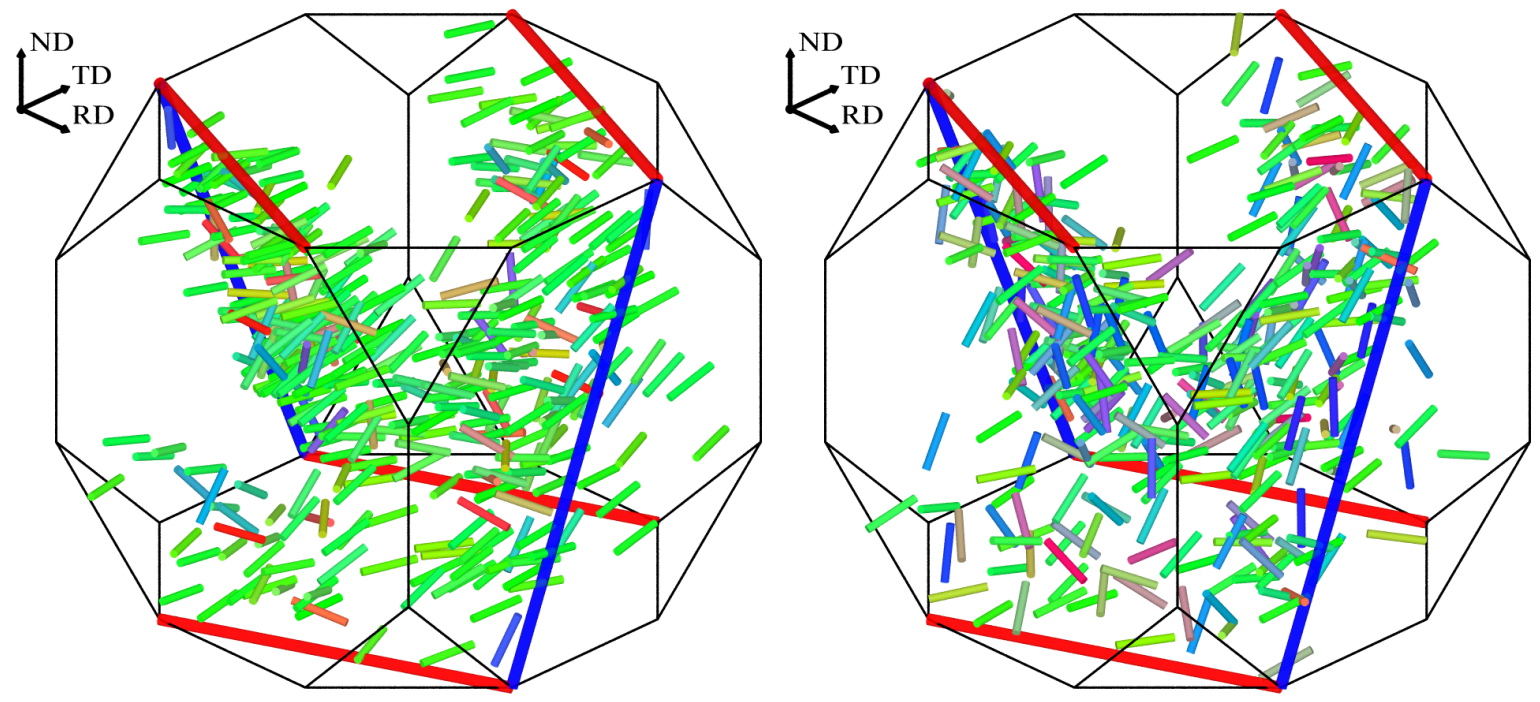

(c)
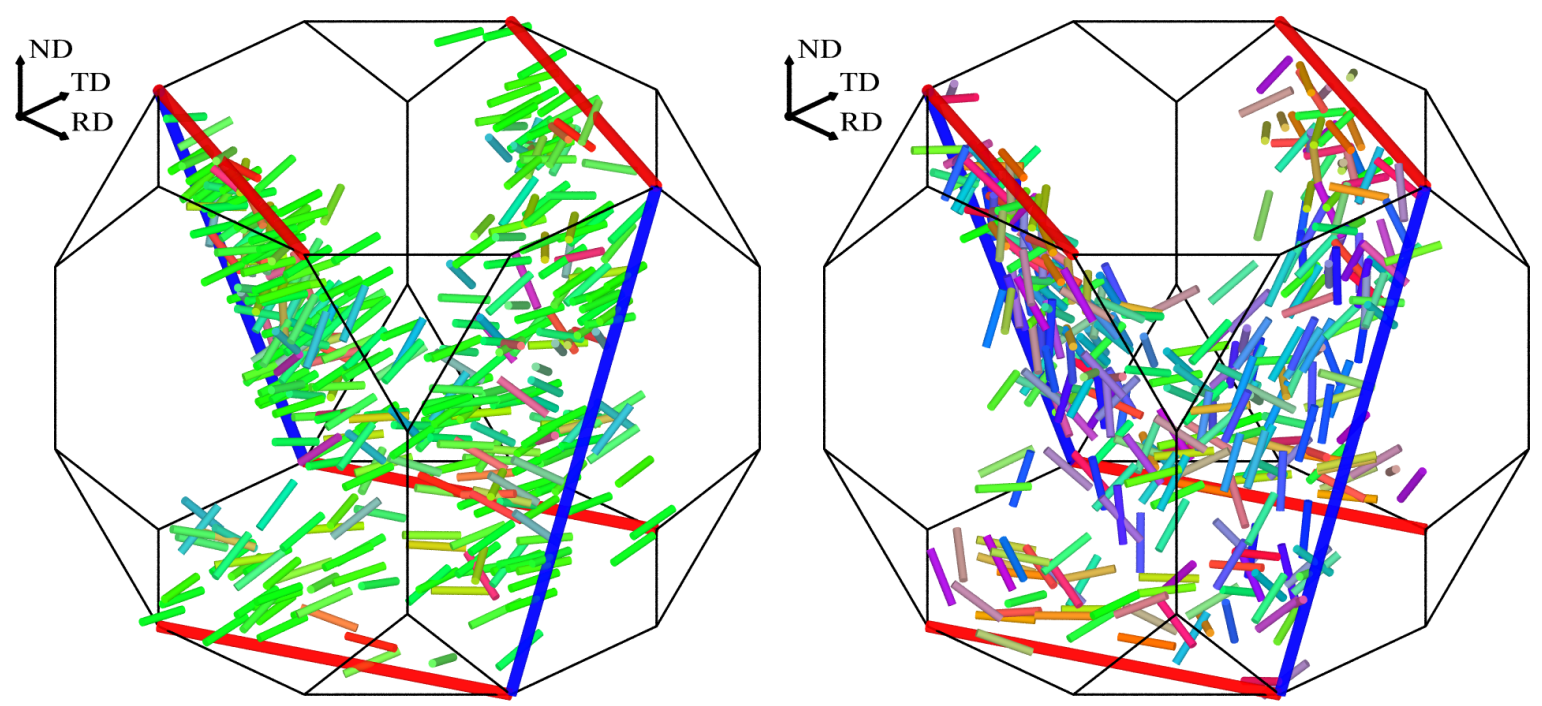

Figure 7: Correlation between the preferential disorientation axes and the average orientations of the grains, illustrated in the Rodrigues fundamental region. For each grain, the preferential disorientation axis is represented as a baton located at the average orientation of the grain. The colour of the axis is related to its direction. (a) $\varepsilon=0.02$, (b) $\varepsilon=$ 0.19 , (c) $\varepsilon=0.42$, (d) $\varepsilon=0.77$ and (e) $\varepsilon=1.2$. Left is experiment and right is simulation. 

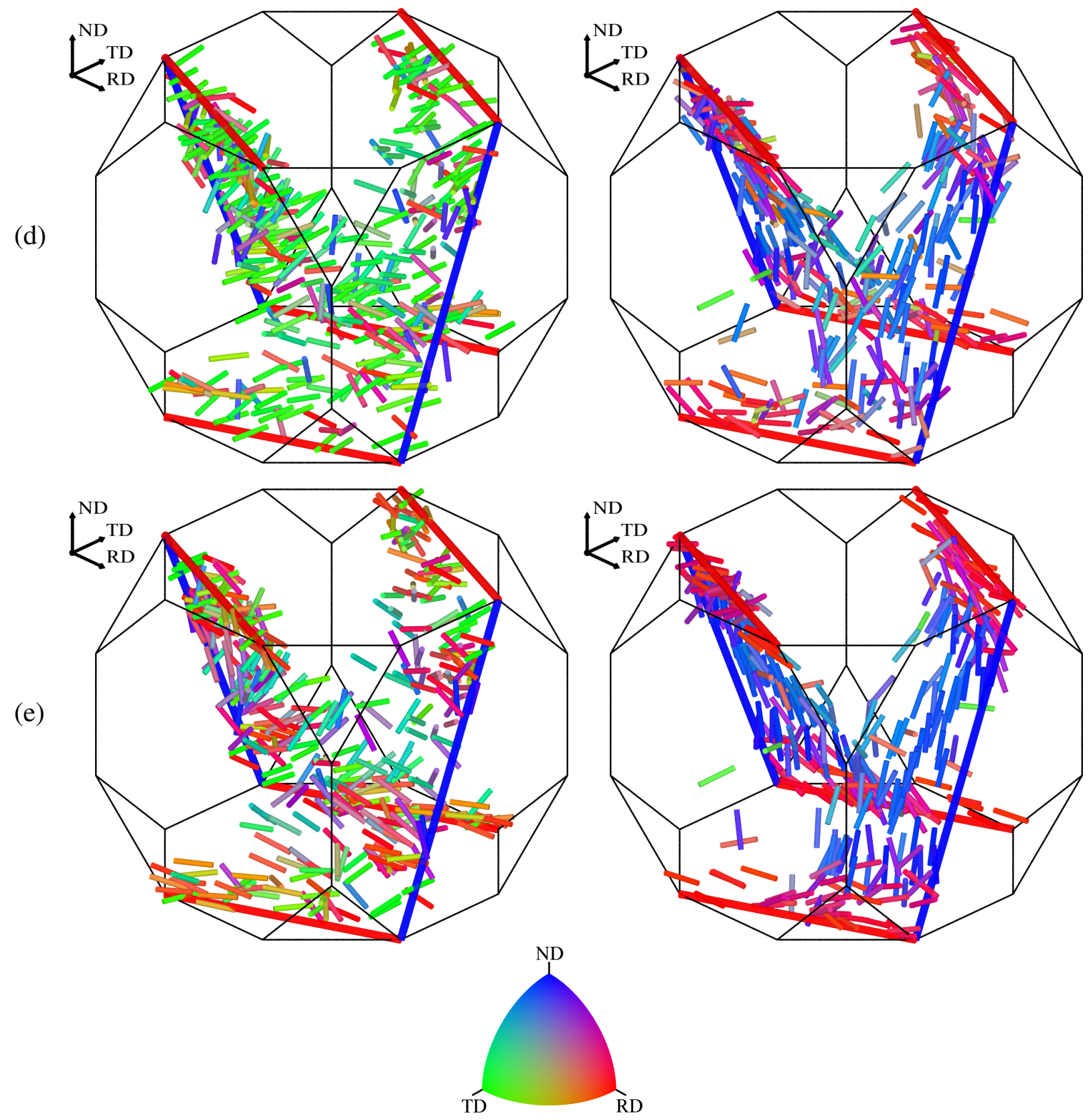

Figure 7: (continued) 


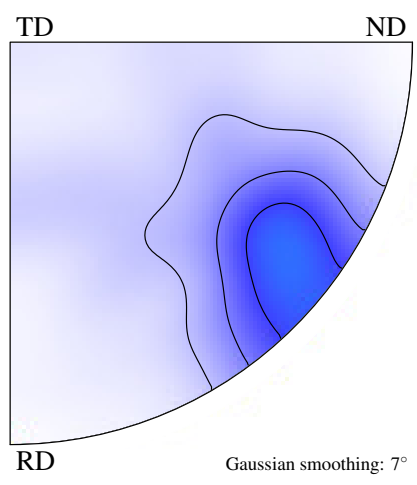

(a)

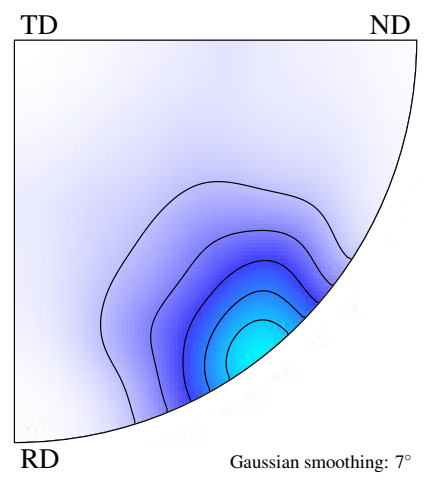

(b)

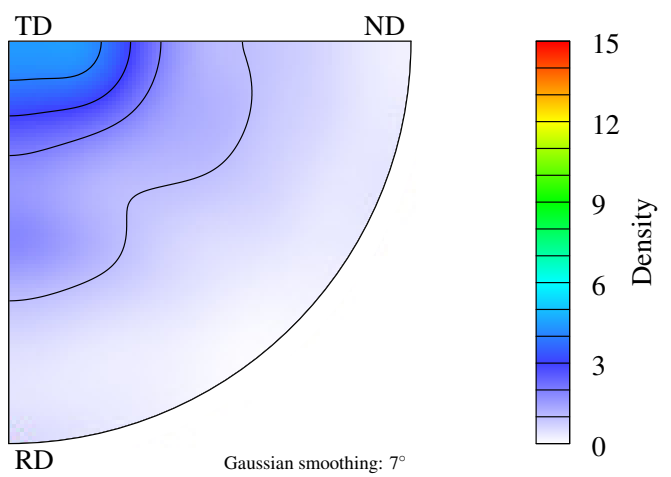

(c)

Figure 8: Geometry of the active slip systems for the 92 grains in simulation, represented as the distributions of (a) $\left|\overline{\dot{\gamma}^{\alpha}}\right| \overline{\mathbf{m}^{\alpha}}$, (b) $\left|\overline{\dot{\gamma}^{\alpha}}\right| \overline{\mathbf{s}^{\alpha}}$ and (c) $\left|\overline{\dot{\gamma}^{\alpha}}\right| \overline{\mathbf{t}^{\alpha}}$ (equal-area projections). 


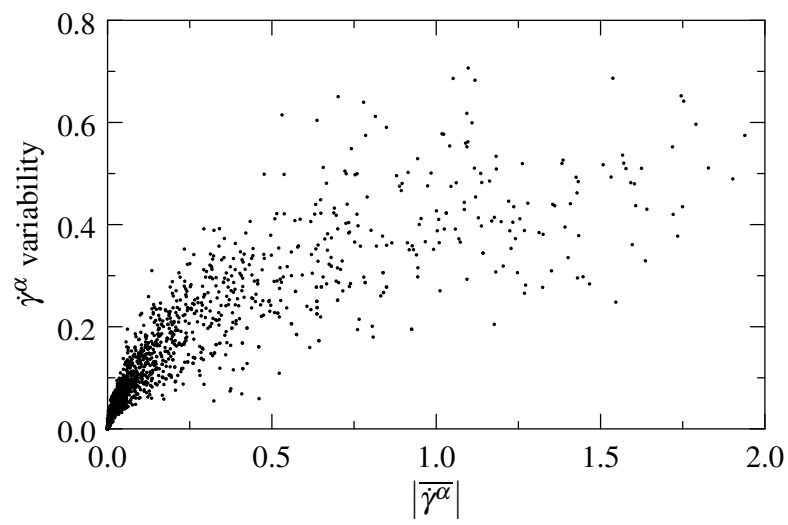

Figure 9: Relation between the slip rate variability and the average slip rate. The data are plotted for the slip systems of the 92 grains in simulation and the variability stands for the standard deviation. Each point comes from a specific slip system of a grain ( 24 points per grain). 


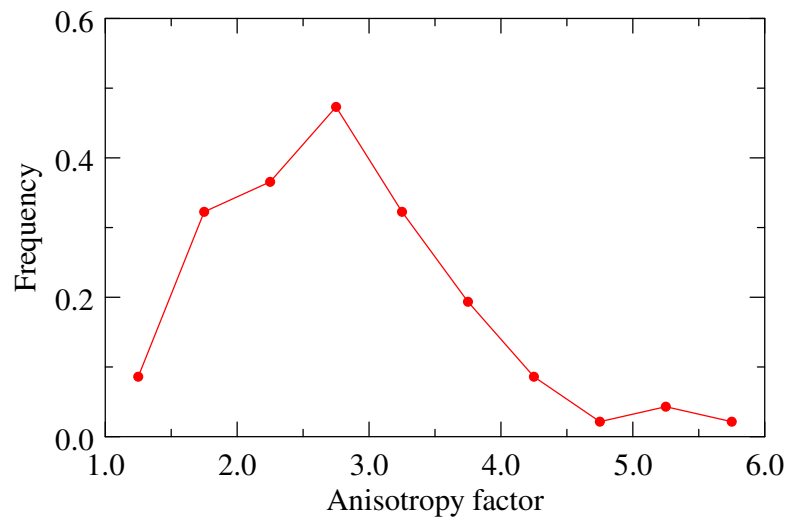

Figure 10: Intra-grain orientation distribution anisotropy factors resulting from an isotropic stress variability. 


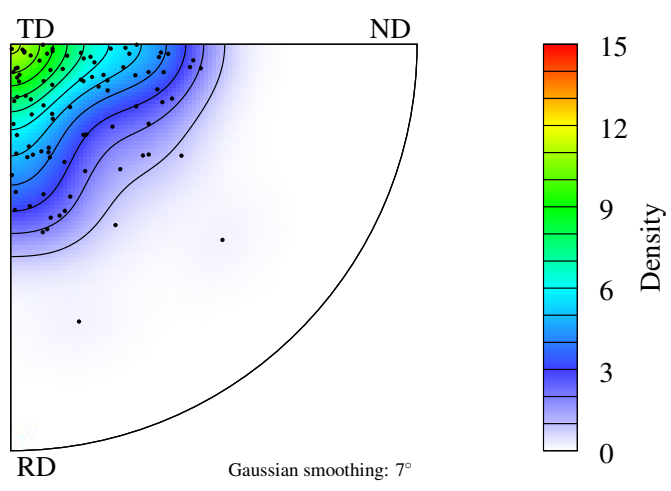

(a)

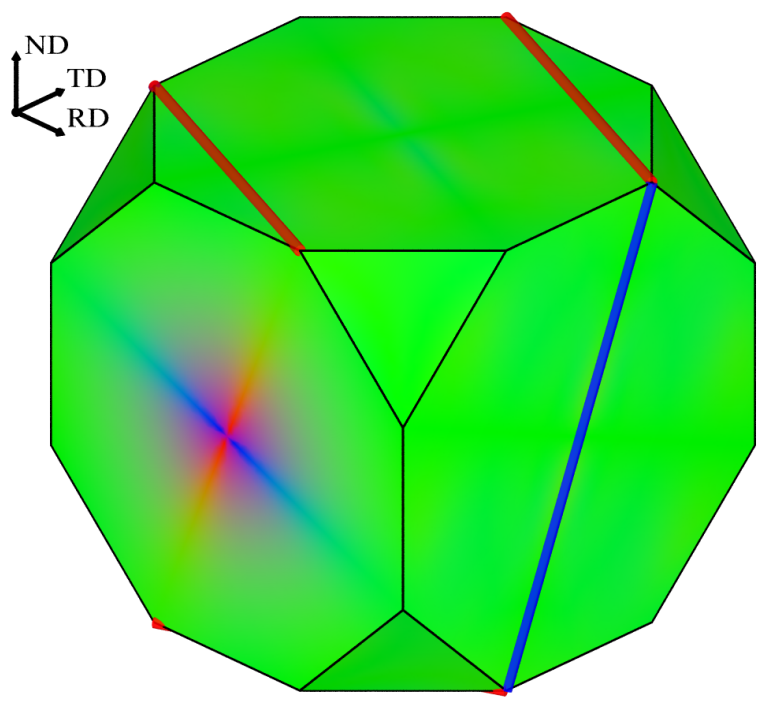

(c)

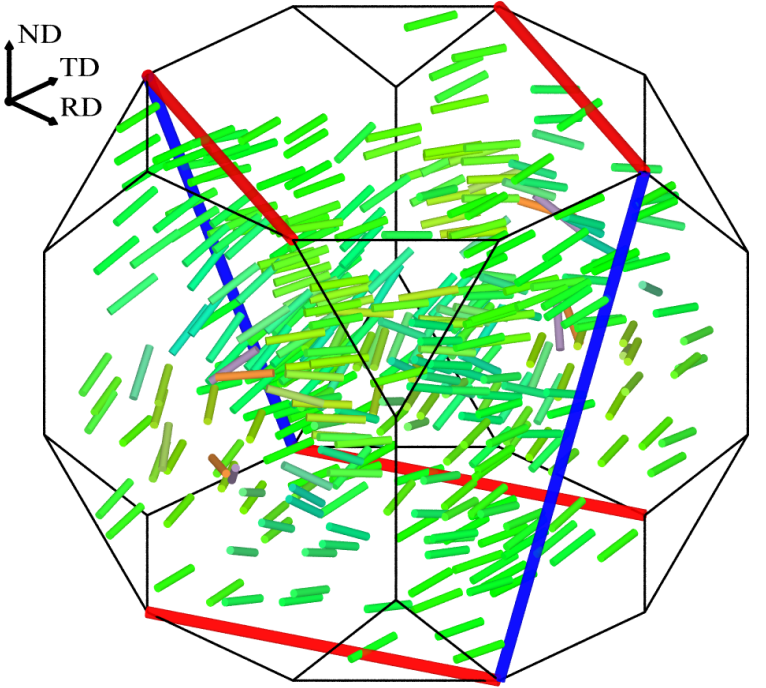

(b)

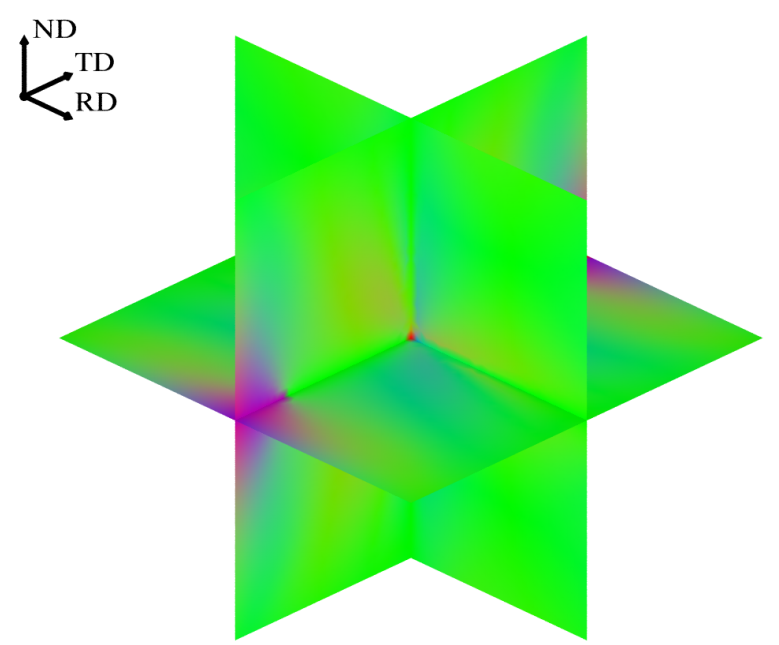

(d)

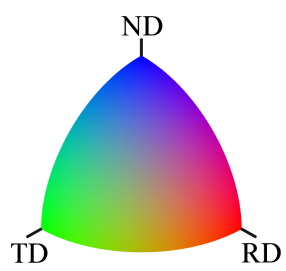

Figure 11: Preferential disorientation axes resulting from an isotropic stress variability shown as (a) distribution over all initial orientations (equal-area projection), (b) correlation with the associated average orientations, (c) distribution over the Rodrigues fundamental region and (d) slices along the sample symmetry planes. 


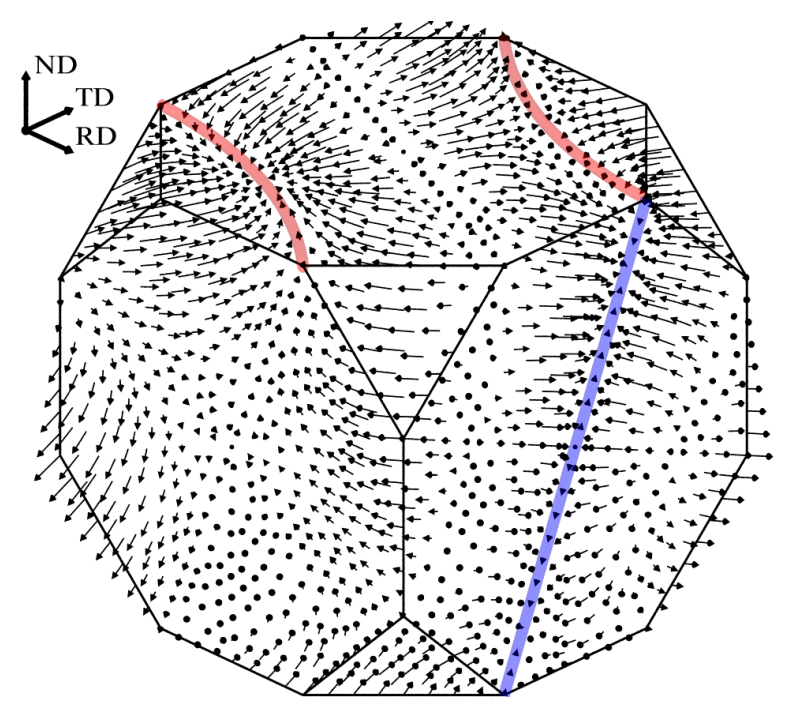

Figure 12: Reorientation velocity field represented on the surface of the Rodrigues fundamental region. Note the convergence of the velocity field about the $\alpha$ and $\beta$ fibres (shown in blue and red, respectively). Compared to Figure 6, the ideal $\beta$ fibre has been bent to match the convergence line of the velocity field. 

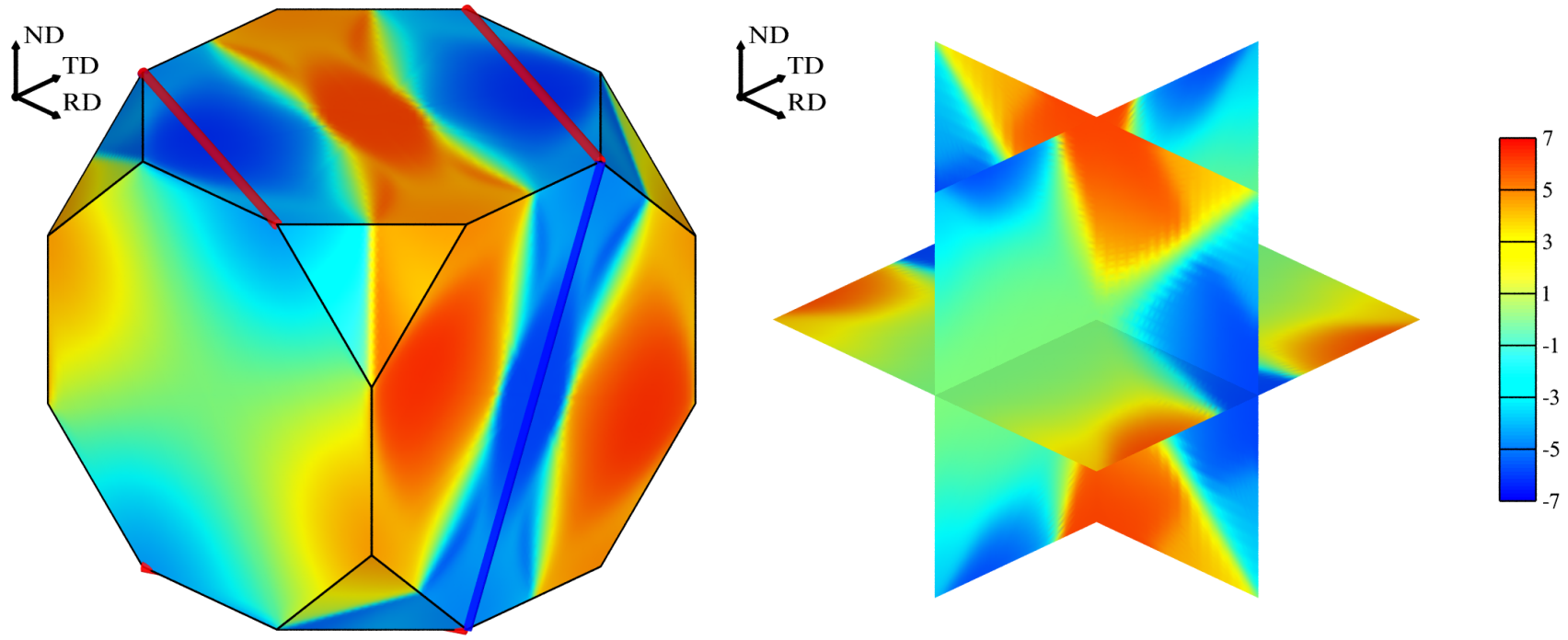

(a)

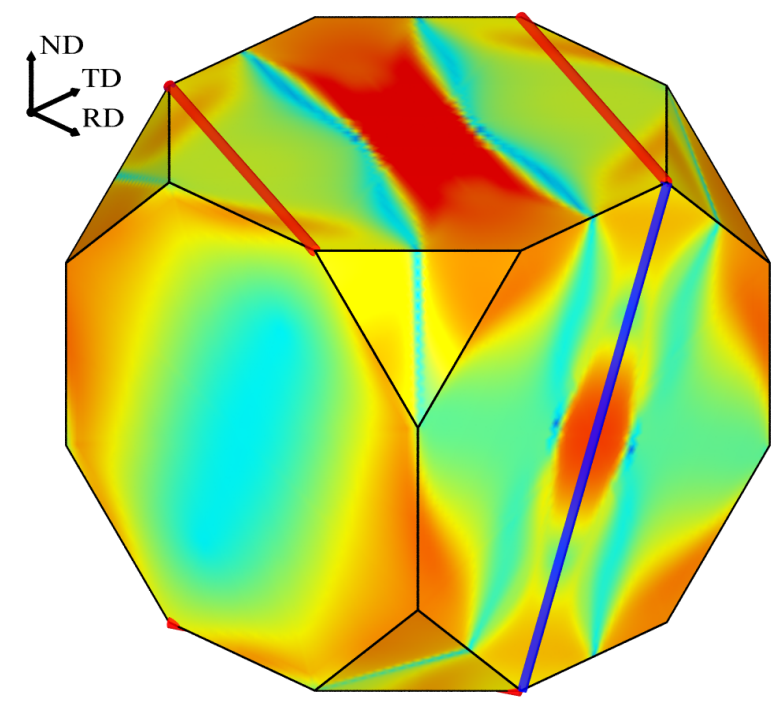

$\bigsqcup_{\mathrm{RD}}^{\mathrm{ND}}$

(b)
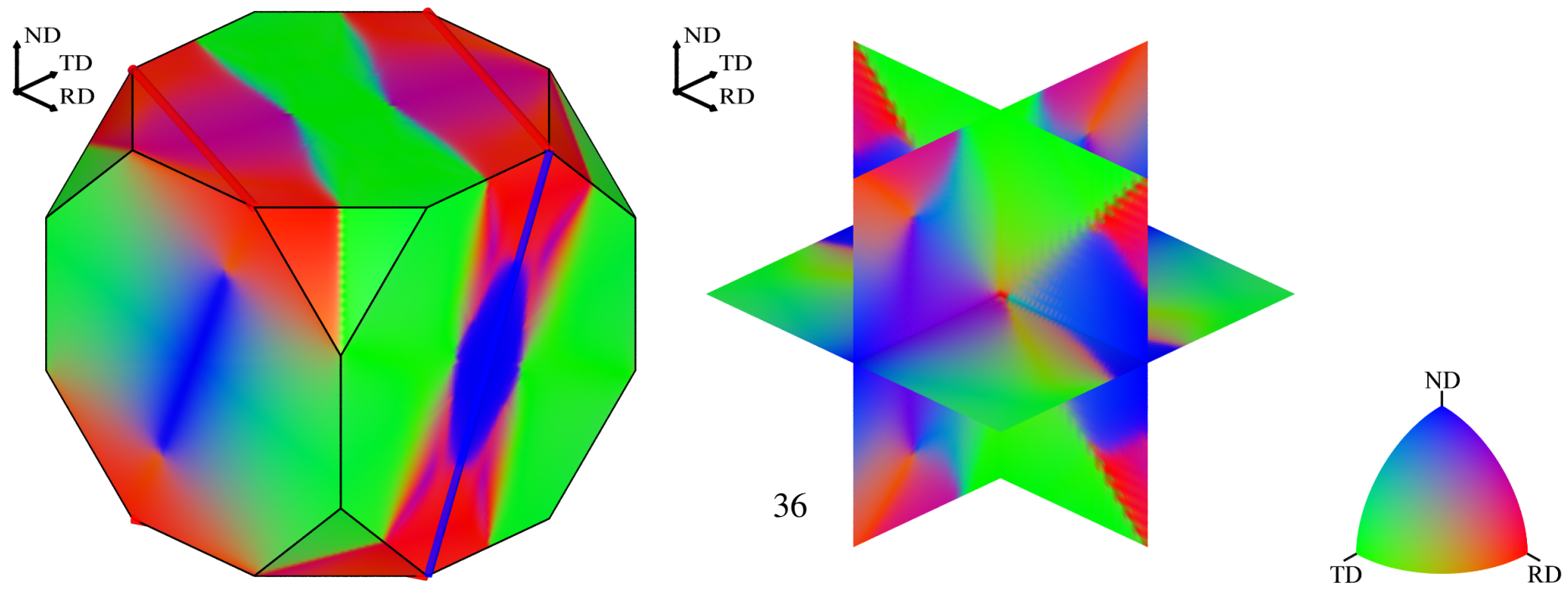

(c)

Figure 13: Properties of the Taylor reorientation velocity field. (a) Divergence, (b) velocity gradient anisotropy and (c) first principal axis (corresponding to the highest principal value). 


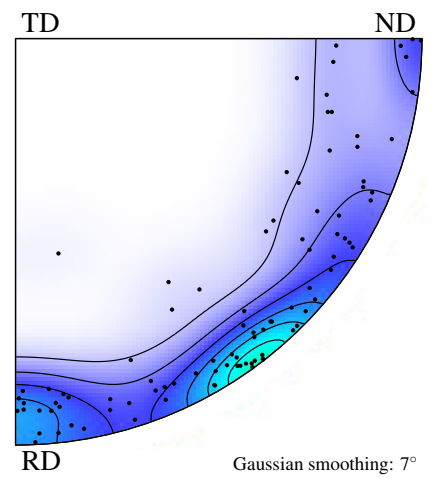

(a)

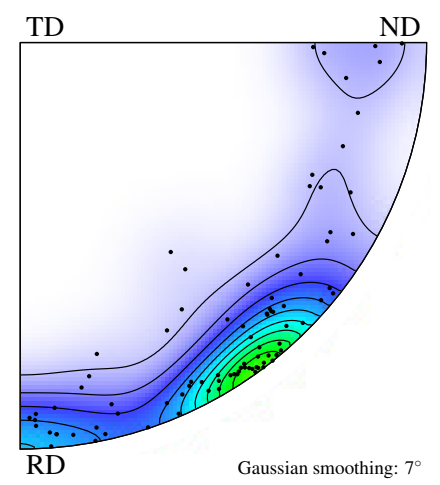

(b)

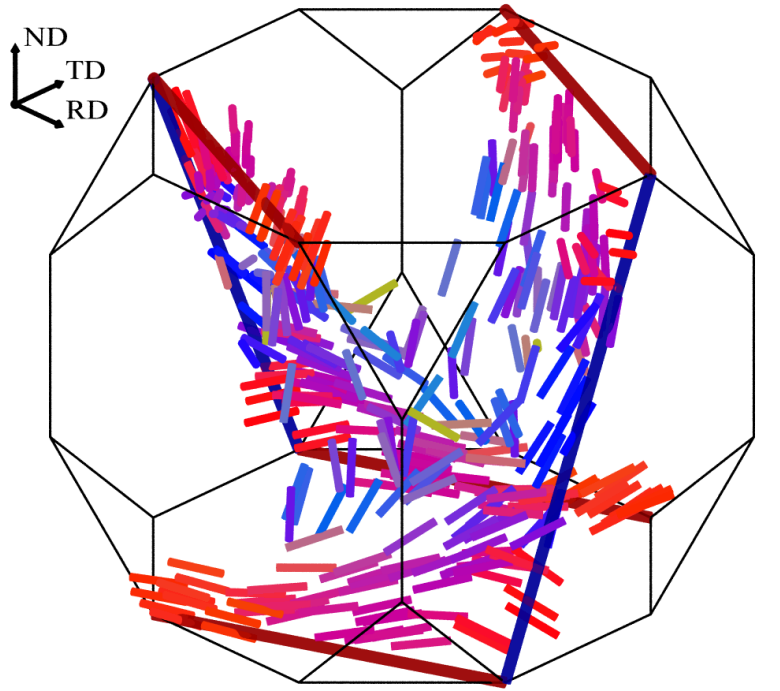

(c)

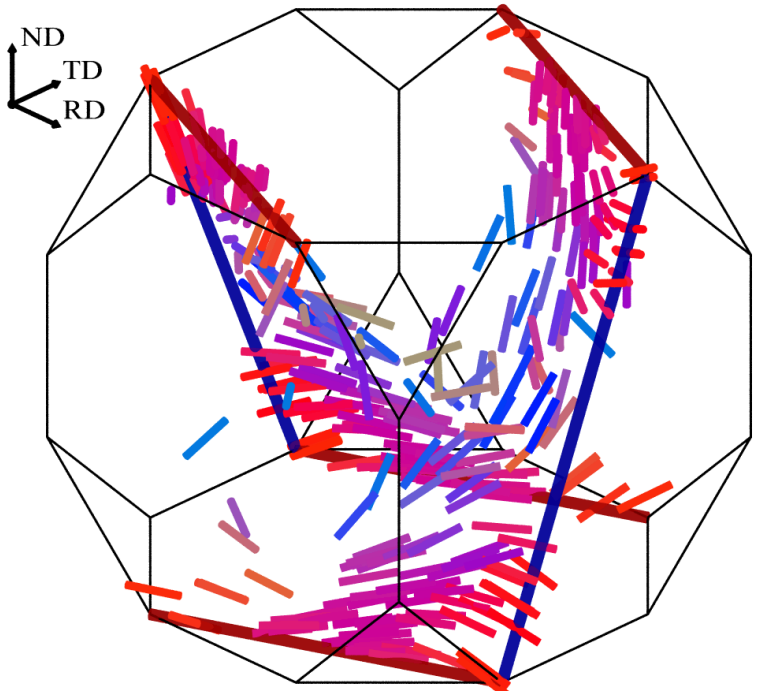

(d)

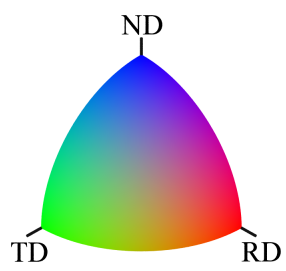

Figure 14: Preferential disorientation axes provided by the Taylor reorientation velocity field shown as distribution over all (a) experimental and (b) simulated final orientations, and correlation with the associated average orientations for (c) experiment and (d) simulation. (a) and (b) are equal-area projections. 


\section{List of Tables}

1 Crystal deformation parameters used in the finite element simulation. The ratios between the slip hardness values of the different systems are taken from Ref. [27].] 39

2 Evolution of the average disorientation angles and anisotropy factors with strain in the experiment and simulation. . . . . . . . . . . . . . . . . 40 


\begin{tabular}{llllcl}
\hline Systems & $\dot{\gamma}_{0}\left(\mathrm{~s}^{-1}\right)$ & $m$ & $h_{0}(\mathrm{MPa})$ & $g_{0}(\mathrm{MPa})$ & $g_{s}(\mathrm{MPa})$ \\
\hline$\{111\}\langle 110\rangle$ & 1 & 0.12 & 4 & 8.0 & 12.0 \\
$\{110\}\langle 110\rangle$ & 1 & 0.12 & 4 & 11.2 & 16.8 \\
$\{100\}\langle 110\rangle$ & 1 & 0.12 & 4 & 7.2 & 10.8 \\
\hline
\end{tabular}

Table 1: Crystal deformation parameters used in the finite element simulation. The ratios between the slip hardness values of the different systems are taken from Ref. [27]. 


\begin{tabular}{lllll}
\hline \multirow{2}{*}{ Strain } & \multicolumn{2}{l}{ Average disorientation angle, $\overline{\boldsymbol{\theta}}\left[{ }^{\circ}\right]$} & \multicolumn{2}{l}{ Anisotropy factor, $\lambda_{a}$} \\
& Experiment & Simulation & Experiment & Simulation \\
\hline 0.19 & 3.5 & 3.3 & 1.91 & 1.62 \\
0.42 & 5.2 & 5.7 & 1.75 & 1.64 \\
0.77 & 6.5 & 8.0 & 1.55 & 1.84 \\
1.20 & 7.2 & 9.9 & 1.49 & 2.13 \\
\hline
\end{tabular}

Table 2: Evolution of the average disorientation angles and anisotropy factors with strain in the experiment and simulation. 